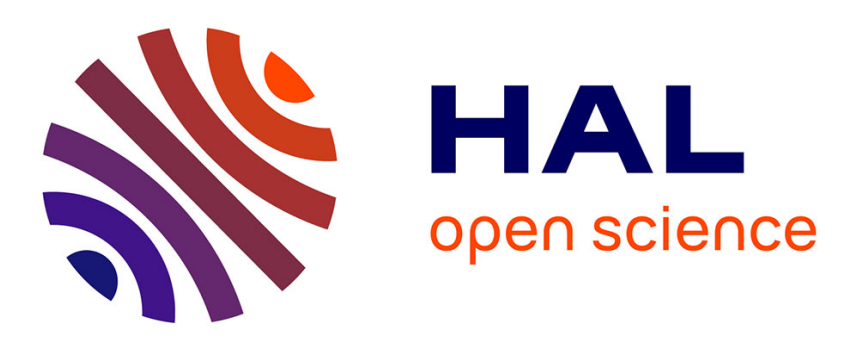

\title{
On the Validation of the Linear Sampling Method in Electromagnetic Inverse Scattering Problems
}

\author{
Francis Collino, M'B. Fares, Houssem Haddar
}

\section{To cite this version:}

Francis Collino, M'B. Fares, Houssem Haddar. On the Validation of the Linear Sampling Method in Electromagnetic Inverse Scattering Problems. [Research Report] RR-4665, INRIA. 2002. inria00071920

\section{HAL Id: inria-00071920 \\ https://hal.inria.fr/inria-00071920}

Submitted on 23 May 2006

HAL is a multi-disciplinary open access archive for the deposit and dissemination of scientific research documents, whether they are published or not. The documents may come from teaching and research institutions in France or abroad, or from public or private research centers.
L'archive ouverte pluridisciplinaire HAL, est destinée au dépôt et à la diffusion de documents scientifiques de niveau recherche, publiés ou non, émanant des établissements d'enseignement et de recherche français ou étrangers, des laboratoires publics ou privés. 
INSTITUT NATIONAL DE RECHERCHE EN INFORMATIQUE ET EN AUTOMATIQUE

\title{
On the Validation of the Linear Sampling Method in Electromagnetic Inverse Scattering Problems
}

\author{
F. Collino - M'B. Fares - H. Haddar
}

$N^{\circ} 4665$

Décembre 2002

THÈME 4 



\title{
On the Validation of the Linear Sampling Method in Electromagnetic Inverse Scattering Problems
}

\author{
F. Collino*†, M'B. Fares* ${ }^{*}$ H. Haddar ${ }^{\dagger}$ \\ Thème 4 - Simulation et optimisation \\ de systèmes complexes \\ Projet Ondes \\ Rapport de recherche $\mathrm{n}^{\circ} 4665$ - Décembre 2002 - 37 pages
}

\begin{abstract}
We present in this study some three dimensional numerical results that validate the use of the Linear Sampling Method as an inverse solver in electromagnetic scattering problems. We recall that this method allows the reconstruction of the shape of an obstacle from the knowledge of multisatic radar data at a fixed frequency. It does not require any a priori knowledge on the physical properties of the scatterer nor any nonlinear optimization scheme. This study contains also some analytical results in the simplified case of a spherical scatterer that somehow make the link between known abstract theoretical results and the numerical scheme. Special attention has been made to point out the influence of the frequency on the inversion accuracy.
\end{abstract}

Key-words: inverse problem, scattering, electromagnetic, Linear Sampling Method, farfield

* CERFACS, 42 Av Coriolis, 31057 Toulouse Cedex 01, France

$\dagger$ INRIA-Rocquencourt 


\section{Sur la validation de la Linear Sampling Method dans les problèmes inverses électromagnétiques}

Résumé : Nous présentons dans ce document quelques résultats de validation de la Linear Sampling Method. Il s'agit d'une méthode rapide d'inversion qui permet de retrouver le support d'un objet à partir de sa réponse radar multi-statique à une fréquence donnée. Nous considérons ici le cas d'ondes électromagnétiques en dimension 3 de l'espace. Les différents résultats numériques présentés sont épaulés par une étude analytique préliminaire dans le cas simplifié d'un objet diffractant sphérique. Cette étude permet entre autre d'établir un lien entre les résultats théoriques connus sur la validité de la méthode et l'algorithme numérique qui lui est associé. Nous mettons l'accent également sur l'influence de la fréquence sur la résolution de la reconstruction.

Mots-clés : problème inverse, diffraction, électromagnétique, Linear Sampling Method, champ lointain 


\section{Introduction}

The Linear Sampling Method (LSM) has gained a growing interest in recent years in inverse scattering problems, due to its effectiveness especially in treating 3-D inverse problems, and also due to its large spectrum of applications. We recall that this algorithm allows the reconstruction of the shape of an obstacle (or a local inhomogeneity) from multistatic data at a fixed frequency. Unlike classical nonlinear methods, it is based on solving independent linear systems and requires no a priori knowledge on the physical properties of the scatterers.

This method has been introduced first in [2] and applied to Maxwell's equations in [1] and [4]. We shall complete these works by numerical and analytical validations of this algorithm focusing our study on the following three points:

1. Analytical study in the case of the sphere. As presented in $[1,4]$, the justification of the LSM uses the concept of nearby solutions, without explaining the link between these nearby solutions and the regularized solution used in the numerical algorithm. The aim of this analytical study is to clarify this point. It also allows us to verify the application of the factorization method introduced by Kirsch [6] in the case of Maxwell's equations. We recall that this method avoids the notion of nearby solution, but its justification in the case of Maxwell's equation is still an open problem.

2. Influence of the frequency. We shall explain how the quality of the LSM reconstructions is frequency dependent. For example, the LSM allows, in principle, simultaneous reconstructions of the shape of disconnected scatterers. However, one observes numerically that the distinction between two different objects is possible only if these two objects are sufficiently far away from each other (respectively to the wavelength of the incident waves). We shall also point out the influence of the frequency on the reconstruction resolution, and show (analytically in the case of the sphere and numerically for complex geometries) how the resolution increases when one increases the frequency.

3. Limited aperture reconstructions. After validating the use of LSM in the case of full aperture data, i.e. we measure all around the obstacle the scattered field that results from incident plane waves coming from all directions, we shall also validate the use of this algorithm when the multistatic data is only available on a limited aperture.

\section{A brief description of the LSM algorithm}

\subsection{The forward problem}

We consider a scatterer that occupies a domain $D \subset \mathbb{R}^{3}$ with bounded and regular boundary $\partial D$. This domain is illuminated by a harmonic electromagnetic incident wave at a frequency $k$. The (dimensionless) electromagnetic field $(E, H)$ satisfies, in an established harmonic regime,

$$
\begin{gathered}
\operatorname{curl} E-i k H=0, \operatorname{curl} H+i k E=0 \text { in } \mathbb{R}^{3} \backslash D \\
\nu \times(E \times \nu)+z_{r} \nu \times H=0 \text { on } \partial D,
\end{gathered}
$$


where $\nu$ is the normal to $\partial D$ directed to the exterior of $D$ and where $z_{r}$ is the relative impedance of the boundary. For physical reasons, this impedance must have a non positive real part. Let us recall that: $z_{r}=0$ corresponds to a perfectly conducting scatterer and $z_{r}=-i k \delta$ corresponds to a coated scatterer by a thin dielectric layer, where $\delta$ depends on both the coating thickness and and the dielectric relative permittivity, [10].

The total electromagnetic field $(E, H)$ is such that

$$
E=E^{i}+E^{s}, H=H^{i}+H^{s},
$$

where $\left(E^{s}, H^{s}\right)$ is the scattered field, satisfying the Silver-Müller radiation condition

$$
\lim _{|x| \rightarrow \infty}\left(H^{s} \times x-|x| E^{s}\right)=0
$$

uniformly with respect to $\hat{x}=x /|x|$, where the incident field $\left(E^{i}, H^{i}\right)$ is a solution of Maxwell's equations in $\mathbb{R}^{3}$. We consider here the cases where these incident fields corresponds to plane waves

$$
\begin{aligned}
& E^{i}(x)=\frac{i}{k} \operatorname{curl} \operatorname{curl} p e^{i k x \cdot d}=i k(\hat{d} \times p) \times d e^{i k x \cdot d} \\
& H^{i}(x)=\operatorname{curl} p e^{i k x \cdot d}=i k \hat{d} \times p e^{i k x \cdot d}
\end{aligned}
$$

where the unitary vector $\hat{d}$ and the vector $p$ denote respectively the direction of propagation and the polarization of the wave.

It is well known that the scattered field $E^{s}$ has the following asymptotic expansion at $\infty$, uniformly with respect to $\hat{x}$,

$$
E^{s}(x)=\frac{e^{i k|x|}}{|x|}\left\{E_{\infty}(\hat{x} ; \hat{d}, p)+O\left(\frac{1}{|x|}\right)\right\}
$$

where $E_{\infty}$ is the electric far field pattern, that analytically depends on $(\hat{x} ; \hat{d}, p)$ and also on $k$. The dependence on $k$ is omitted since it is not taken into account by the LSM. However, as mentioned before, the quality of the results depends on this frequency.

\subsection{The inverse problem and the LSM}

The inverse problem we are interested in is the reconstruction of the domain $D$ from the knowledge of $E_{\infty}(\hat{x}, \hat{d}, p)$ for all $(\hat{x}, \hat{d}, p) \in \Omega \times \Omega \times \mathbb{R}^{3}$, where $\Omega$ denotes the unit sphere. This is the full aperture case. The limited aperture case corresponds to the knowledge of $E_{\infty}(\hat{x}, \hat{d}, p)$ for all $(\hat{x}, \hat{d}, p) \in \Gamma \times \Gamma \times \mathbb{R}^{3}$, where $\Gamma$ is a non empty open subset of $\Omega$. The generalization to this case is obtained by simply substituting $\Gamma$ to $\Omega$ in all the following.

Let us begin by introducing the far field operator that constitutes the basis of the LSM. This operator is defined on the Hilbert space $T^{2}(\Omega)$;

$$
T^{2}(\Omega):=\left\{g: \Omega \rightarrow \mathbb{C}^{3} \mid g \in L^{2}(\Omega), g \cdot \hat{x}=0 \text { for } \hat{x} \in \Omega\right\} .
$$


by: $F: T^{2}(\Omega) \rightarrow T^{2}(\Omega)$ such that

$$
(F g)(\hat{x}):=\int_{\Omega} E_{\infty}(\hat{x} ; d, g(d)) d s(d), \quad \hat{x} \in \Omega,
$$

Let us recall that $F$ is a linear and compact operator on $T^{2}(\Omega)$.

Now, consider a parameter $z \in D$ and an electromagnetic dipole $\left(E_{e}, H_{e}\right)$ of momentum $q \in \mathbb{R}^{3}$ located at $z$,

$$
\begin{aligned}
& E_{e}(x ; z, q):=\operatorname{curl}_{x} q \Phi(x, z) \\
& H_{e}(x ; z, q):=\frac{1}{i k} \operatorname{curl}_{x} \operatorname{curl}_{x} q \Phi(x, z),
\end{aligned}
$$

where $\Phi$ is the fundamental solution to the Helmholtz equation, given by

$$
\Phi(x, z):=\frac{1}{4 \pi} \frac{e^{i k|x-z|}}{|x-z|}, x \neq z .
$$

The LSM is based on seeking, for fixed $q$ and $z$, a function $g(\cdot, z, q) \in T^{2}(\Omega)$ solution of

$$
(F g(\cdot ; z, q))(\hat{x})=E_{e, \infty}(\hat{x} ; z, q):=\frac{i k}{4 \pi}(\hat{x} \times q) e^{-i k \hat{x} \cdot z},
$$

where $E_{e, \infty}$ is nothing but the far field pattern associated to $E_{e}$. In general, this equation does not have any solution (as shown for example in the case of a sphere), however one can prove the existence of a nearby solution $g_{\varepsilon}(\cdot ; z, q) \in T L^{2}(\Omega)$, i.e. satisfying $\| F g_{\varepsilon}(\cdot ; z, q)-$ $E_{e, \infty}(\cdot ; z, q) \| \leq \varepsilon$, where $\varepsilon$ is a small parameter independent of $(z, q)$, such that

$$
\lim _{\substack{z \rightarrow \partial D \\ z \in D}}\left\|g_{\varepsilon}(\cdot ; z, q)\right\|_{L^{2}(\Omega)}=\infty .
$$

Furthermore, (formally) $\left\|g_{\varepsilon}(\cdot, z, q)\right\|=\infty$ for $z \in \mathbb{R}^{3} \backslash D$ (see $[1,4,5]$ ). Consequently the $L^{2}$ norm of $g_{\varepsilon}(\cdot, z, q)$ can be used as an indicator of the domain $D$ that we want to reconstruct. This is roughly speaking the idea of the LSM.

The main weak point in this justification is of course the lack of constructive method to determine this nearby solution. In practice, we use a regularization process to compute a nearby solution to (10); we consider a Tikhonov regularization by seeking $g_{\eta}(\cdot, z, q) \in T^{2}(\Omega)$ solution of

$$
\left(\eta+F^{*} F\right) g_{\eta}(\cdot, z, q)=F^{*} E_{e, \infty}(\cdot ; z, q)
$$

where $\eta$ is the regularization parameter. The link between this regularized solution and the nearby solution will be investigated in the case of a sphere.

Another variant of the LSM is the factorization method introduced by Kirsch in the acoustic case [6]. This method is based on solving the following equation,

$$
\sqrt{|F|} g(\cdot, z, q)=E_{e, \infty}(\cdot ; z, q) .
$$


We shall prove in the special case of a coated sphere that this equation is solvable in $T^{2}(\Omega)$ if and only if $z$ is in the interior of the sphere. The generalization of this result to other geometries is still under study.

\section{Analytical computations in the case of a sphere}

We consider in this section the case where the scatterer is a sphere of a radius $a$.

\subsection{Analytical expression of the far fields}

The derivation of an analytic expression of the far fields $E_{\infty}(\cdot ; \hat{d}, p)$ relies on the decomposition of the incident and scattered fields in terms of Debye's potentials (see [11] page 259, for instance). The details of the calculations are given in Appendix A. To give the result, let us precise some notations. We introduce the functions

$$
u_{n}^{m}(x)=j_{n}(k|x|) Y_{n}^{m}(\hat{x}), \quad v_{n}^{m}(x)=h_{n}^{(1)}(k|x|) Y_{n}^{m}(\hat{x}), \quad x=|x| \hat{x},
$$

$n=1, \cdots, \infty, m=-n, \cdots, n$, where $\left(Y_{n}^{m}\right)$ denotes the set of orthonormal spherical harmonics, $\left(j_{n}\right)$ denotes the set of spherical Bessel functions and $\left(h_{n}^{(1)}\right)$ denotes the set of spherical Hankel functions of the first kind. We then define the sets $\left(M_{n}^{m}\right)$ and $\left(N_{n}^{m}\right)$ of respectively entire and radiating solutions to Maxwell's equations by

$$
M_{n}^{m}(x)=\operatorname{curl}\left(x u_{n}^{m}(x)\right), \quad N_{n}^{m}(x)=\operatorname{curl}\left(x v_{n}^{m}(x)\right) .
$$

We also define the vector spherical harmonics of order $n$

$$
U_{n}^{m}(\hat{x})=\frac{1}{\sqrt{n(n+1)}} \operatorname{Grad} Y_{n}^{m}(\hat{x}), \quad V_{n}^{m}(\hat{x})=\frac{1}{\sqrt{n(n+1)}} \hat{x} \times \operatorname{Grad}_{n}^{m}(\hat{x}) .
$$

We recall that the set of vector spherical harmonics form an orthonormal basis of $T^{2}(\Omega)$. We finally recall the definitions of the Ricatti-Bessel functions

$$
\psi_{n}(t)=t j_{n}(t), \quad \zeta_{n}^{(1)}(t)=t h_{n}^{(1)}(t)
$$

With the help of these definitions, the electric far field pattern resulting from the scattering of a plane wave by an impedant sphere has the following expansion (see Lemma A.4)

$$
E_{\infty}(\hat{x} ; \hat{d}, p)=4 \pi \sum_{n=1}^{\infty} \sum_{m=-n}^{n} u_{n}\left(\overline{U_{n}^{m}}(\hat{d}) \cdot p\right) U_{n}^{m}(\hat{x})+v_{n}\left(\overline{V_{n}^{m}}(\hat{d}) \cdot p\right) V_{n}^{m}(\hat{x})
$$


where the coefficients $u_{n}$ and $v_{n}$ depends on both $k a$ and the impedance $z_{r}$. They are given by

$$
\left\{\begin{array}{l}
u_{n}=-\frac{i \psi_{n}{ }^{\prime}(k a)+z_{r} \psi_{n}(k a)}{i \zeta_{n}^{(1)^{\prime}}(k a)+z_{r} \zeta_{n}^{(1)}(k a)}\left(:=u_{n}\left(k a, z_{r}\right)\right), \\
v_{n}=-\frac{\psi_{n}(k a)+i z_{r} \psi_{n}^{\prime}(k a)}{\zeta_{n}^{(1)}(k a)+i z_{r} \zeta_{n}^{(1)^{\prime}}(k a)}\left(:=v_{n}\left(k a, z_{r}\right)\right) .
\end{array}\right.
$$

We remark that these coefficients are well defined for each $n$ since the denominators cannot vanish if $\Re e z_{r} \leq 0$. The proof of this statement is the following: assume for example that

$$
i \zeta_{n}^{(1){ }^{\prime}}(k a)+z_{r} \zeta_{n}^{(1)}(k a)=0 .
$$

Due to the Nicholson formula, $\zeta_{n}^{(1)}(t)$ never vanishes for $t>0$ and therefore the previous equality is equivalent to

$$
i \zeta_{n}^{(1)^{\prime}}(k a) \overline{\zeta_{n}^{(1)}(k a)}+z_{r}\left|\zeta_{n}^{(1)}(k a)\right|^{2}=0
$$

which implies

$$
(k a)^{2}\left(j_{n}^{\prime}(k a) y_{n}(k a)-j_{n}(k a) y_{n}^{\prime}(k a)\right)+\Re e\left(z_{r}\left|\zeta_{n}^{(1)}(k a)\right|^{2}\right)=0,
$$

where $y_{n}$ denotes the Neumann spherical function of order $n$. One recognizes the Wronksian of spherical Bessel functions in the previous equality, that gives

$$
-1+\Re e\left(z_{r}\right)\left|\zeta_{n}^{(1)}(k a)\right|^{2}=0
$$

which cannot hold if $\Re e z_{r} \leq 0$. The proof that $i z_{r} \zeta_{n}^{(1)^{\prime}}(k a)+\zeta_{n}^{(1)}(k a) \neq 0$ is identical.

We also remark that if $\Re e z_{r} \neq 0$ the coefficients $u_{n}$ and $v_{n}$ never vanish, since all the zeros of the Bessel functions are simple. This means in particular, as explained in the sequel, that the far field operator is injective in that case. However, when $\Re e z_{r}=0$, it may happen that some coefficients vanish for some particular values of $k$. This situation corresponds the the existence of resonant frequencies for which the far field operator is therefore no longer injective.

In the following we assume that $z_{r}$ and $k$ are such that $u_{n} \neq 0$ and $v_{n} \neq 0$.

Expansion (13) implies that the far field operator is diagonal in the Riesz basis $\left(U_{n}^{m}, V_{n}^{m}\right)_{n, m}$ : let $g \in T^{2}(\Omega)$, then

$$
g(\hat{d})=\sum_{n=1}^{\infty} \sum_{m=-n}^{n} g_{u, n}^{m} U_{n}^{m}(\hat{d})+g_{v, n}^{m} V_{n}^{m}(\hat{d}) \quad \hat{d} \in \Omega,
$$

with coefficients

$$
g_{u, n}^{m}=\int_{\Omega} g(\hat{d}) \cdot \overline{U_{n}^{m}}(\hat{d}) d s(\hat{d}), \quad g_{v, n}^{m}=\int_{\Omega} g(\hat{d}) \cdot \overline{V_{n}^{m}}(\hat{d}) d s(\hat{d}) .
$$


Simple calculations then show

$$
(F g)(\hat{x})=4 \pi \sum_{n=1}^{\infty} \sum_{m=-n}^{n}\left(u_{n} g_{u, n}^{m}\right) U_{n}^{m}(\hat{x})+\left(v_{n} g_{v, n}^{m}\right) V_{n}^{m}(\hat{x}) .
$$

Therefore $4 \pi u_{n}$ and $4 \pi v_{n}$ are nothing but the eigenvalues of the compact operator $F$ respectively associated the eigenfunctions $U_{n}^{m}$ and $V_{n}^{m}$. Before solving the far field equation we shall first give a sight of the behavior of the far field operator by plotting the graph of these eigenvalues as functions of $n$.

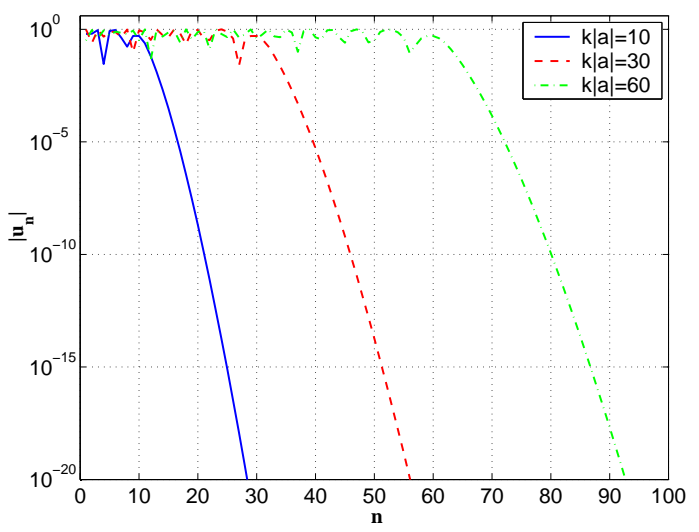

Figure 1: $\left|u_{n}\right|$ versus $n$ for $z_{r}=0$

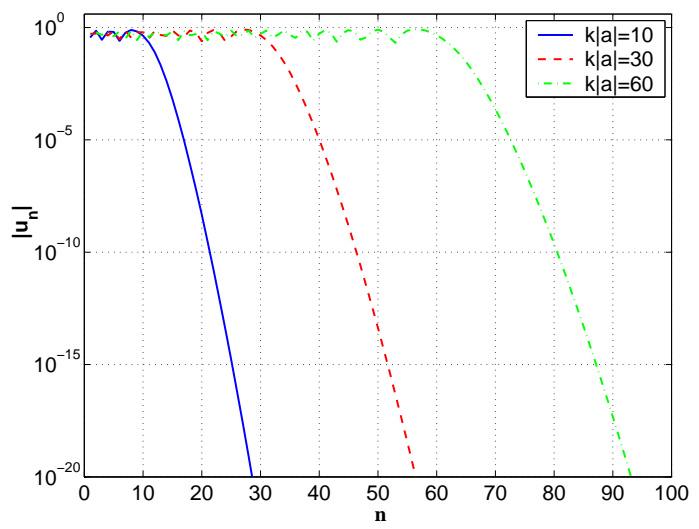

Figure 3: $\left|u_{n}\right|$ versus $n$ for $z_{r}=-1+i$

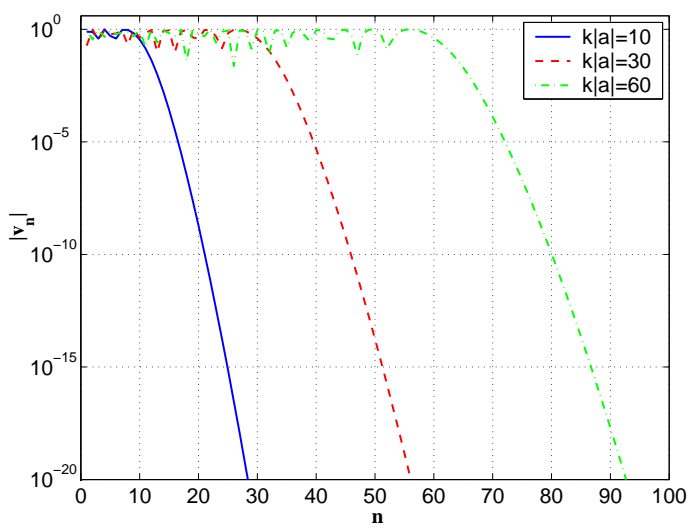

Figure 2: $\left|v_{n}\right|$ versus $n$ for $z_{r}=0$

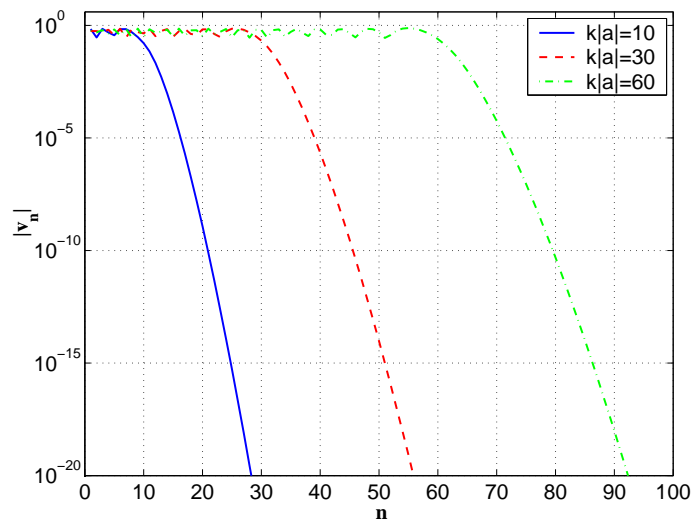

Figure 4: $\left|v_{n}\right|$ versus $n$ for $z_{r}=-1+i$

We observe that the modulus of these eigenvalues remains close to 1 for $n<k|a|$ but decreases more than exponentially for $n>k|a|$. We can also see this by studying the 
asymptotic behavior of these eigenvalues with respect to $n$. From the following well known asymptotic expansions, see [3] page 28 ,

$$
j_{n}(t)=\frac{t^{n}}{(2 n+1) ! !}\left(1+O\left(\frac{1}{n}\right)\right), h_{n}^{(1)}(t)=\frac{(2 n-1) ! !}{i t^{n+1}}\left(1+O\left(\frac{1}{n}\right)\right),
$$

(where we used the notation $(2 n+1) ! !:=1 \cdot 3 \cdots(2 n+1)$ ), as well as the identities

$$
\begin{aligned}
\psi_{n}{ }^{\prime}(t) & =\frac{t}{2 n+1}\left((n+1) j_{n-1}(t)-n j_{n+1}(t)\right), \\
\zeta_{n}^{(1)^{\prime}}(t) & =\frac{t}{2 n+1}\left((n+1) h_{n-1}^{(1)}(t)-n h_{n+1}^{(1)}(t)\right),
\end{aligned}
$$

it is readily seen that

$$
\left|u_{n}\right| \sim\left|v_{n}\right| \sim \frac{(k a)^{2 n+1}}{[(2 n-1) ! !]^{2}(2 n+1)} .
$$

We notice in particular that the asymptotic behavior is independent of $z_{r}$, which can be also seen by comparing Figure 1 to Figure 3 and Figure 2 to Figure 4

\subsection{Far field equation and regularized solution}

We now consider the far field equation: $g(\cdot ; z, q) \in T^{2}(\Omega)$ such that

$$
(F g(\cdot ; z, q))(\hat{x})=E_{e, \infty}(\hat{x} ; z, q)=\frac{i k}{4 \pi}(\hat{x} \times q) e^{-i k \hat{x} \cdot z} .
$$

Let us set

$$
\begin{gathered}
E_{e, \infty}(\hat{x} ; z, q)=\sum_{n=1}^{\infty} \sum_{m=-n}^{n} \alpha_{n}^{m}(z, q) U_{n}^{m}(\hat{x})+\beta_{n}^{m}(z, q) V_{n}^{m}(\hat{x}), \\
g(\hat{x} ; z, q)=\sum_{n=1}^{\infty} \sum_{m=-n}^{n} g_{u, n}^{m}(z, q) U_{n}^{m}(\hat{x})+g_{v, n}^{m}(z, q) V_{n}^{m}(\hat{x}) .
\end{gathered}
$$

We deduce from previous subsection that if the solution to the far field equation exists then it is given by

$$
g_{u, n}^{m}(z, q)=\alpha_{n}^{m}(z, q) /\left(4 \pi u_{n}\right) \quad \text { and } \quad g_{v, n}^{m}(z, q)=\beta_{n}^{m}(z, q) /\left(4 \pi v_{n}\right) .
$$

But as we shall prove now, this solution does not belong to $T^{2}(\Omega)$.

Lemma 3.1 Let $\left(g_{u, n}^{m}, g_{v, n}^{m}\right)_{n, m}$ be given by (17), then

$$
\|g(\cdot ; z, q)\|^{2}=\sum_{n=1}^{\infty} \sum_{m=-n}^{n}\left|g_{u, n}^{m}(z, q)\right|^{2}+\left|g_{v, n}^{m}(z, q)\right|^{2}=\infty, \quad \text { for all } z \neq 0 \text { and } q \neq 0 .
$$


Proof. It is proved in Appendix A, Lemma A.1 that

$$
\alpha_{n}^{m}(z, q)=\frac{(-i)^{n}}{\sqrt{n(n+1)}}\left(i k \overline{M_{n}^{m}}(z) \cdot q\right) \text { and } \beta_{n}^{m}(z, q)=\frac{(-i)^{n}}{\sqrt{n(n+1)}}\left(\operatorname{curl} \overline{M_{n}^{m}}(z) \cdot q\right) \text {, }
$$

or equivalently, by setting $z=|z| \hat{z}$,

$$
\begin{aligned}
& \alpha_{n}^{m}(z, q)=\frac{(-i)^{n+1}}{|z|} \psi_{n}(k|z|) \overline{V_{n}^{m}}(\hat{z}) \cdot q \\
& \beta_{n}^{m}(z, q)=\frac{(-i)^{n}}{|z|}\left(\psi_{n}{ }^{\prime}(k|z|) \overline{U_{n}^{m}}(\hat{z}) \cdot q-j_{n}(k|z|) \sqrt{n(n+1)} \overline{Y_{n}^{m}}(\hat{z})(\hat{z} \cdot q)\right) .
\end{aligned}
$$

From the addition theorem for spherical harmonic

$$
(2 n+1) P_{n}(\hat{x} \cdot \hat{y})=4 \pi \sum_{m=-n}^{n} Y_{n}^{m}(\hat{x}) \overline{Y_{n}^{m}}(\hat{y})
$$

where $P_{n}$ denotes the Legendre polynomial. From $\overline{Y_{n}^{m}}=Y_{n}^{-m}$, we readily deduce that

$$
\sum_{m=-n}^{n}\left|Y_{n}^{m}(\hat{z})\right|^{2}=(2 n+1) /(4 \pi) \text { and } \sum_{m=-n}^{n} \operatorname{Grad}_{n}^{m}(\hat{z}) \overline{Y_{n}^{m}}(\hat{z})=\sum_{m=-n}^{n} U_{n}^{m}(\hat{z}) \overline{Y_{n}^{m}}(\hat{z})=0
$$

On the other hand, it is also shown in Appendix A, Lemma A.5 that,

$$
\sum_{m=-n}^{n}\left|V_{n}^{m}(\hat{d}) \cdot p\right|^{2}=\sum_{m=-n}^{n}\left|U_{n}^{m}(\hat{d}) \cdot p\right|^{2}=\frac{2 n+1}{8 \pi}\|\hat{d} \times p\|^{2} .
$$

Identities (21) and (22) yield then

$$
\begin{aligned}
\sum_{m=-n}^{n}\left|\alpha_{n}^{m}(z, q)\right|^{2} & =\left(\frac{2 n+1}{8 \pi|z|^{2}}\right) \psi_{n}(k|z|)^{2}\|\hat{z} \times q\|^{2} \\
\sum_{m=-n}^{n}\left|\beta_{n}^{m}(z, q)\right|^{2} & =\frac{2 n+1}{8 \pi|z|^{2}}\left(\psi_{n}{ }^{\prime}(k|z|)^{2}\|\hat{z} \times q\|^{2}+2 n(n+1) j_{n}(k|z|)^{2}(\hat{z} \cdot q)^{2}\right) .
\end{aligned}
$$

Let us set

$$
\alpha_{n}(z, q)=\frac{1}{k}\left(\sum_{m=-n}^{n}\left|\alpha_{n}^{m}(z, q)\right|^{2}\right)^{\frac{1}{2}} \text { and } \beta_{n}(z, q)=\frac{1}{k}\left(\sum_{m=-n}^{n}\left|\beta_{n}^{m}(z, q)\right|^{2}\right)^{\frac{1}{2}},
$$

we therefore have

$$
\|g(\cdot ; z, q)\|^{2}=\frac{k^{2}}{(4 \pi)^{2}} \sum_{n=1}^{\infty} \frac{\left|\alpha_{n}(z, q)\right|^{2}}{\left|u_{n}\right|^{2}}+\frac{\left|\beta_{n}(z, q)\right|^{2}}{\left|v_{n}\right|^{2}}
$$


From (15) and the asymptotic expansions

$$
j_{n}(t)=\frac{t^{n}}{(2 n+1) ! !}\left(1+O\left(\frac{1}{n}\right)\right), \psi_{n}{ }^{\prime}(t)=\frac{1}{2} \frac{t^{n}}{(2 n-1) ! !}\left(1+O\left(\frac{1}{n}\right)\right),
$$

one deduces that

$$
\begin{aligned}
& \frac{\left|\alpha_{n}(z, q)\right|^{2}}{\left|u_{n}\right|^{2}}=\frac{2 n+1}{8 \pi(k|z|)^{2}}\left((2 n-1) ! ! \frac{(k|z|)^{n+1}}{(k a)^{2 n+1}}\right)^{2}\|\hat{z} \times q\|^{2}\left(1+O\left(\frac{1}{n}\right)\right), \\
& \frac{\left|\beta_{n}(z, q)\right|^{2}}{\left|v_{n}\right|^{2}}=\frac{2 n+1}{8 \pi(k|z|)^{2}}\left((2 n+1) ! ! \frac{(k|z|)^{n}}{(k a)^{2 n+1}}\right)^{2}\left(\frac{\|\hat{z} \times q\|^{2}}{4}+\frac{(\hat{z} \cdot q)^{2}}{2}\right)\left(1+O\left(\frac{1}{n}\right)\right) .
\end{aligned}
$$

We then conclude that $\|g(\cdot ; z, q)\|^{2}=\infty$, for all $z \neq 0$ and $q \neq 0$.

Regularization by truncation. We remark though, that the blowing up of the norm of $g$ is only due to high order eigenvalues of $F$ that are very small (see figures 1-4). In practice (numerical or experimental), these eigenvalues are not taken into account, and therefore we only solve (16) restricted to

$$
V_{N}(\Omega):=\operatorname{span}\left\{\left(U_{n}^{m}, V_{n}^{m}\right) ; n=1, \cdots, N ; m=-n, \cdots, n\right\},
$$

where the truncation level $N$ is chosen so that $\left|u_{n}\right|$ and $\left|v_{n}\right|$ are of the same order as numerical errors. This truncated solution should be close to the numerical solution computed with a regularization scheme used by the LSM. Let us see now how this truncated solution can be used to determine the value of $a$.

In figures $5-6$ we plot the norm of the truncated solution $g_{N}(\cdot, z, q)$ given by

$$
\left\|g_{N}(\cdot ; z, q)\right\|^{2}=\frac{1}{(4 \pi)^{2}} \sum_{n=1}^{N} \frac{\left|\alpha_{n}(z, q)\right|^{2}}{\left|u_{n}\right|^{2}}+\frac{\left|\beta_{n}(z, q)\right|^{2}}{\left|v_{n}\right|^{2}} .
$$

where $N$ is chosen such that $\left|u_{N}\right|<10^{-3}$ and $\left|v_{N}\right|<10^{-3}$.

We remark that $\left\|g_{N}\right\|$ is very large in the region $|z| \gg a$ and is relatively small for $|z| \ll a$. So, the value of $a$ can be determined as the mid point of the transition region. As observed in Figures 5 and 6 , this rather ambiguous way of determining $a$ can be very accurate for high frequencies $(k>12)$. However, for low frequencies $(k=3)$, it is does not seem possible to determine with acceptable accuracy the size of the sphere.

As it will be confirmed later, the linear sampling method reconstruction is then frequency dependent, and larger is the frequency, the more accurate is the reconstruction.

Remark 3.1 As shown by figures 1-4 if one increases the frequency, one needs to increase the cut off range $N$. This means that one needs to increase the number of discretization points in numerical inversions (see section 4 below) (the number of points corresponds in practice to the number of measurements). Therefore, a kind of balance between precision and measurement numbers should be considered. 

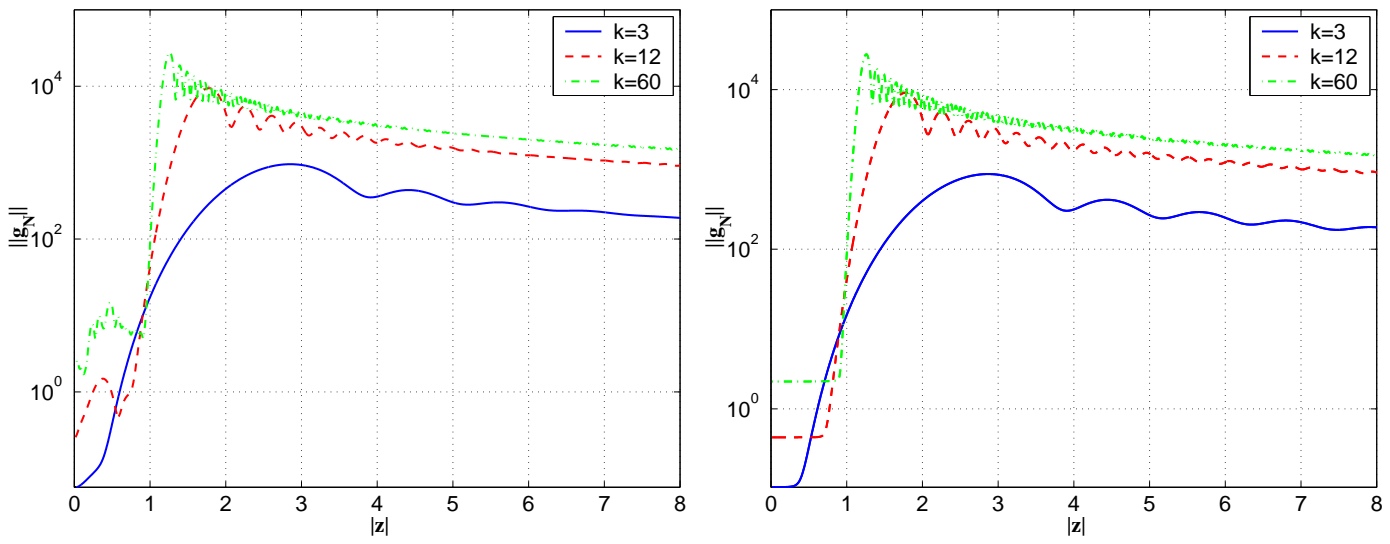

Figure 5: $\left\|g_{N}\right\|$ with respect to $|z|$ for $a=1$ Figure 6: $\left\|g_{N}\right\|$ with respect to $|z|$ for $a=1$ and $z_{r}=0$. Truncation level $=10^{-3}$. and $z_{r}=-1+i$. Truncation level $=10^{-3}$.

In order to explain the behavior of the truncated solution norm, we plot in the following the behavior of $\left|\alpha_{n}(z, q)\right|$ and $\left|\beta_{n}(z, q)\right|$ with respect to $n$ for different values of $k|z|$.

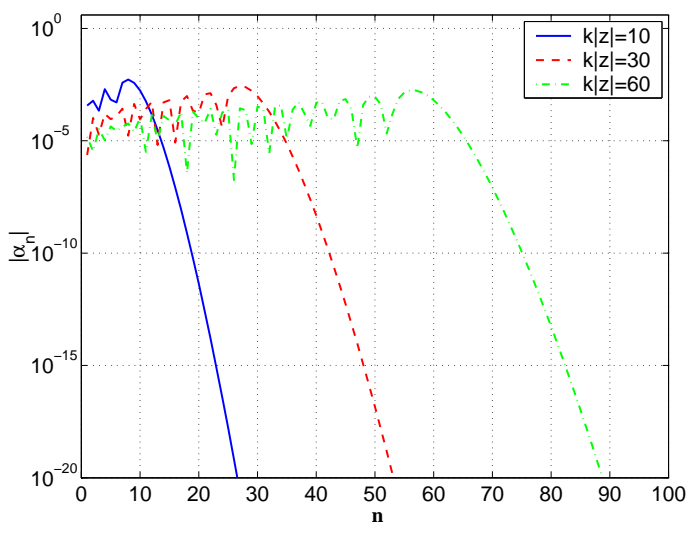

Figure 7: $\left|\alpha_{n}\right|$ versus $n$

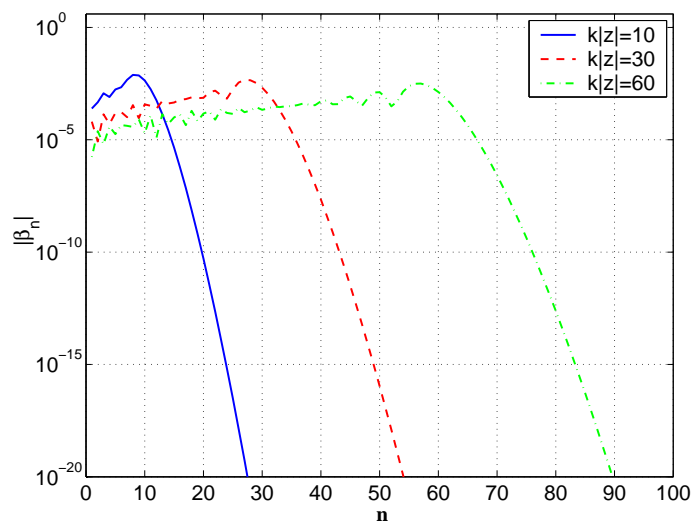

Figure 8: $\left|\beta_{n}\right|$ versus $n$

We observe that these values start to decrease only for $n>k|z|$. Therefore, if we compare with the behavior of $\left|u_{n}\right|$ and $\left|v_{n}\right|$ (see figures 1-4), the norm of $g_{N}$ should be large if $|z|>a$ due to the contribution of the terms with index $k a<n<k|z|$. 


\subsection{The factorization method}

A. Kirsch proposed a variant of the LSM that avoids the notion of nearby solution. His method consists in replacing the operator $F$ by $K:=\sqrt{|F|}\left(\right.$ or $K:=\left(F^{*} F\right)^{\frac{1}{4}}$, when $F$ is normal). In the case of the sphere, $F$ is diagonal in the Riesz basis $\left(U_{n}^{m}, V_{n}^{m}\right)_{n, m}$ with eigenvalues $4 \pi\left(u_{n}, v_{n}\right)$, therefore $K$ is also diagonal in the Riesz basis $\left(U_{n}^{m}, V_{n}^{m}\right)_{n, m}$ with eigenvalues $\sqrt{4 \pi}\left(\sqrt{\left|u_{n}\right|}, \sqrt{\left|v_{n}\right|}\right)$. The solution $g(\cdot, z, q) \in T^{2}(\Omega)$ to the problem

$$
(K g(\cdot ; z, q))(\hat{x})=E_{e, \infty}(\hat{x} ; z, q) .
$$

is given by

$$
g(\hat{x} ; z, q)=\sum_{n=1}^{\infty} \sum_{m=-n}^{n} g_{u, n}^{m}(z, q) U_{n}^{m}(\hat{x})+g_{v, n}^{m}(z, q) V_{n}^{m}(\hat{x})
$$

where

$$
g_{u, n}^{m}(z, q)=\alpha_{n}^{m}(z, q) / \sqrt{\left|4 \pi u_{n}\right|} \text { and } g_{v, n}^{m}(z, q)=\beta_{n}^{m}(z, q) / \sqrt{\left|4 \pi v_{n}\right|} .
$$

We therefore have

$$
\|g(\cdot ; z, q)\|^{2}=\frac{k^{2}}{4 \pi} \sum_{n=1}^{\infty} \frac{\left|\alpha_{n}(z, q)\right|^{2}}{\left|u_{n}\right|}+\frac{\left|\beta_{n}(z, q)\right|^{2}}{\left|v_{n}\right|}
$$

where $\alpha_{n}$ and $\beta_{n}$ are given by (24). From the asymptotic behavior

$$
\begin{aligned}
& \frac{\left|\alpha_{n}(z, q)\right|^{2}}{\left|u_{n}\right|}=\frac{1}{8 \pi k|z|}\left(\frac{|z|}{a}\right)^{2 n+1}\|\hat{z} \times q\|^{2}\left(1+O\left(\frac{1}{n}\right)\right), \\
& \frac{\left|\beta_{n}(z, q)\right|^{2}}{\left|v_{n}\right|}=\frac{(2 n+1)^{2}}{8 \pi k a(k|z|)^{2}}\left(\frac{|z|}{a}\right)^{2 n}\left(\frac{\|\hat{z} \times q\|^{2}}{4}+\frac{(\hat{z} \cdot q)^{2}}{2}\right)\left(1+O\left(\frac{1}{n}\right)\right),
\end{aligned}
$$

one see that the solution exists if and only if $|z|<a$, which gives the desired characterization of the sphere.

However, in practice and as it is the case for the $F$ version, we only solve (26) restricted to

$$
V_{N}(\Omega):=\operatorname{span}\left\{\left(U_{n}^{m}, V_{n}^{m}\right) ; n=1, \cdots, N ; m=-n, \cdots, n\right\},
$$

where the truncation level $N$ is chosen such that $\left|u_{n}\right|$ and $\left|v_{n}\right|$ are less than or equal to a given precision $\epsilon$. Figures 9 and 10 display the norm of the truncated solution $g_{N}$ that corresponds to $\epsilon=10^{-3}$, for respectively $z_{r}=0$ and $z_{r}=-1+i$ and for three different values of $k$. In both cases we observe that the contrast between the regions $|z|>a$ and $|z|<a$ deteriorates rapidly for large $|z|$. To obtain a contrast comparable to that of Figures 5 and 6 , one should have more accuracy on the coefficients: see for instance Figures 11 and 12 where $\varepsilon=10^{-6}$. In that sense one may expect that the factorization method to be more sensitive to the noise level than the LSM. However, as predicted by the theory, the norm of the truncated solution starts to increase only for $|z|>a$. This is not the case of the LSM. 

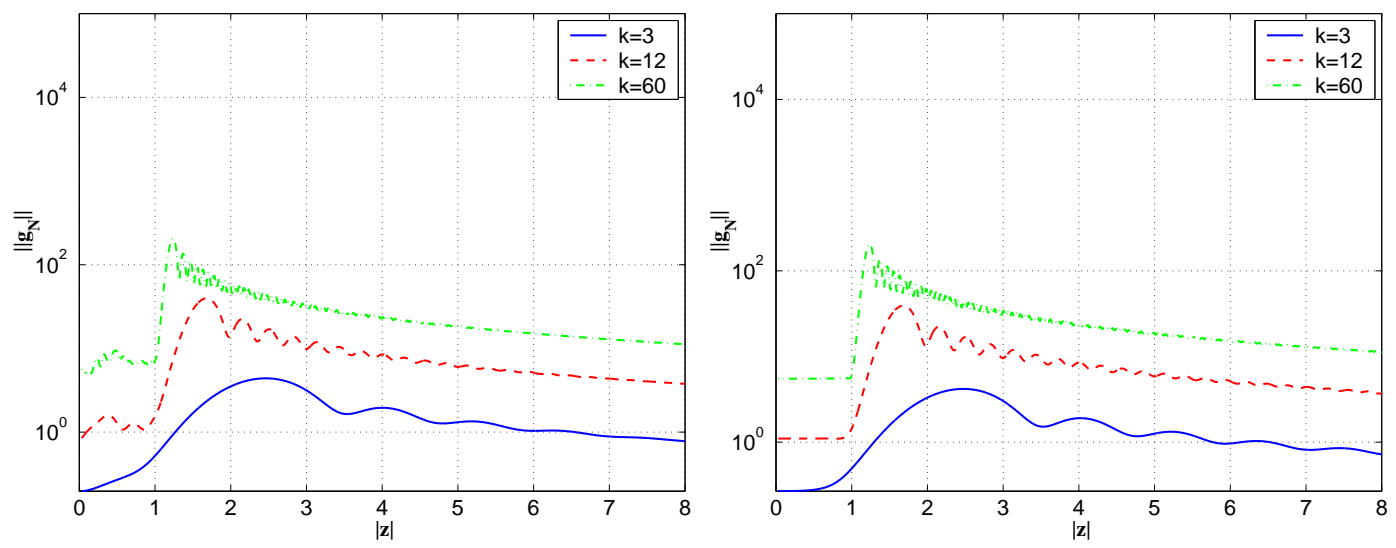

Figure 9: $\left\|g_{N}\right\|$ with respect to $|z|$ for $a=1$ Figure 10: $\left\|g_{N}\right\|$ with respect to $|z|$ for $a=1$ and $z_{r}=0$. Precision $\epsilon=10^{-3}$. and $z_{r}=-1+i$. Precision $\epsilon=10^{-3}$.
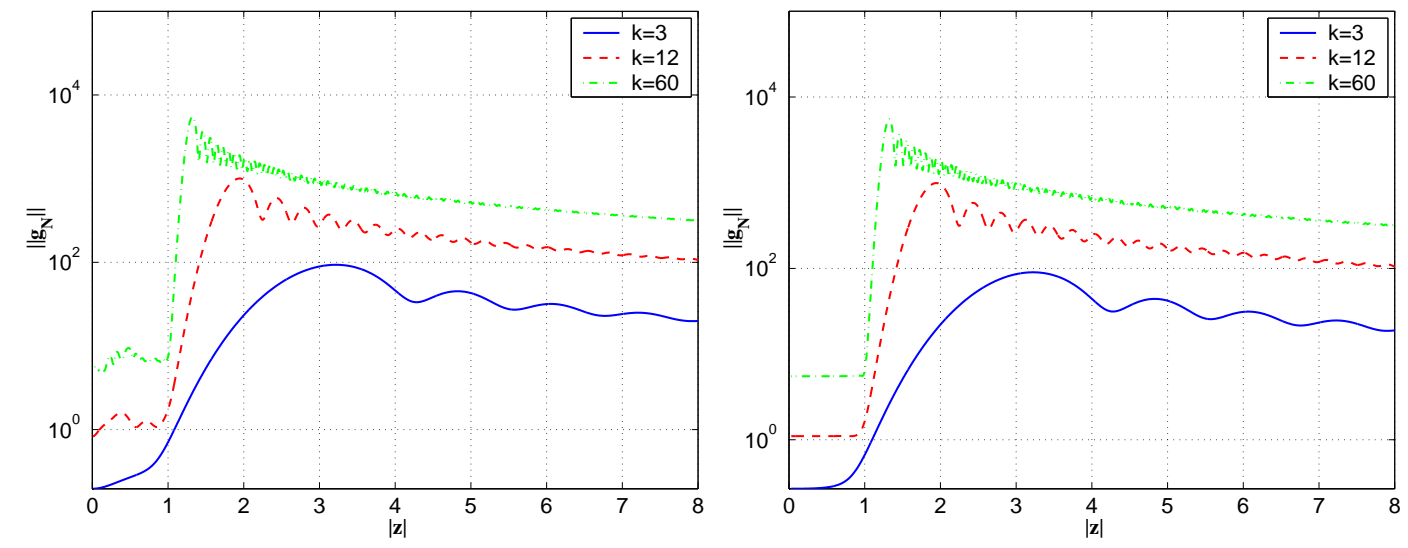

Figure 11: $\left\|g_{N}\right\|$ with respect to $|z|$ for $a=1$ Figure 12: $\left\|g_{N}\right\|$ with respect to $|z|$ for $a=1$ and $z_{r}=0$. Precision $\epsilon=10^{-6}$. and $z_{r}=-1+i$. Precision $\epsilon=10^{-6}$. 


\section{Numerical algorithm}

\subsection{Discrete far field equation}

We adopt here the same discretization scheme of the far field equation (10) as the one used in [1]. We consider a uniform triangular meshing of the unit sphere $\Omega$ (or $\Gamma$, in the case of limited aperture) containing $N$ vertices $\left(\hat{x}_{i}\right)_{1 \leq i \leq N}$. These vertices will serve as directions for the plane incident waves as well as degrees of freedom for the discrete solution of the far field equation.

Let $\hat{p}$ be some unit vector in $\mathbb{R}^{3}$ satisfying $\hat{p} \times \hat{x}_{i} \neq 0$ for all $\hat{x}_{i}$. We define the two orthogonal polarizations

$$
\hat{p}_{j}^{\theta}=\left(\hat{p} \times \hat{x}_{j}\right) /\left|\hat{p} \times \hat{x}_{j}\right| \text { and } \hat{p}_{j}^{\varphi}=\hat{p} \times\left(\hat{x}_{j} \times \hat{p}\right) /\left|\hat{p} \times\left(\hat{x}_{j} \times \hat{p}\right)\right|,
$$

then set

$$
F_{i, j}^{\theta}=E_{\infty}\left(\hat{x}_{i}, \hat{x}_{j}, \hat{p}_{j}^{\theta}\right) \quad \text { and } \quad F_{i, j}^{\varphi}=E_{\infty}\left(\hat{x}_{i}, \hat{x}_{j}, \hat{p}_{j}^{\varphi}\right) .
$$

We construct a continuous approximation of the solution $g(\cdot, z, q)$, linear at each triangle, whose degrees of freedom are its values at the nodes $\left(\hat{x}_{i}\right)_{1 \leq i \leq N}$. The nodal values are denoted by $\left(g_{j}(z, q)\right)_{1 \leq j \leq N}$. Since each vector $g_{j}(z, q)$ is tangent to $\Omega$, we can set

$$
g_{j}(z, q)=g_{j}^{\theta}(z, q) p_{j}^{\theta}+g_{j}^{\varphi}(z, q) p_{j}^{\varphi} .
$$

The integral equation (10) can be transformed at the discrete level into the following linear system of $2 N$ unknowns $\left(g_{j}^{\theta}, g_{j}^{\varphi}\right)$,

$$
\sum_{j=1}^{N} \omega_{j}\left(g_{j}^{\theta}(z, q) F_{i, j}^{\theta}+g_{j}^{\varphi}(z, q) F_{i, j}^{\varphi}\right)=E_{e, \infty}\left(\hat{x}_{i}, z, q\right), \quad \text { for all } i,
$$

where the weights $\omega_{j}$ are linked to the quadrature formulas used in evaluating the integrals over the mesh triangles. Let us remark that, for fixed index $i$, the vectorial equation (30) can be split into two independent scalar equations. System (30) is then a $2 N \times 2 N$ linear system that can be written into the synthetic form

$$
\mathcal{F} G(z, q)=E_{e, \infty}(z, q),
$$

where $\mathcal{F}$ is a $2 N \times 2 N$ matrix that is independent of the parameters $z$ and $q, G(z, q)$ is the unknown vector, whose $\ell^{2}$ norm is expected to be large when $z$ is outside $D$ and finally $E_{e, \infty}(z, q)$ represents the right hand side constructed from the far fields of electromagnetic dipoles.

\subsection{Synthetic generation of the far fields}

We have used CESC to create our synthetic data. CESC is a solver for electromagnetic scattering problems developed at CERFACS. The perfectly conducting case is treated by 
solving the Electric Field Integral Equation, whose unknown is $J=(\nu \times H)_{\left.\right|_{\partial D}}$, the electric current flowing on the surface of the scatterer, [8]. The numerical procedure is based upon a meshing of the surface with triangles and the use of Raviart-Thomas's finite elements of lowest degree, conforming for $H^{-\frac{1}{2}}\left(\operatorname{Div}, \partial D_{h}\right)$. It leads to

Find $J_{h}$ in $V_{h}$ such that

$$
\begin{gathered}
\int_{\partial D_{h}} \int_{\partial D_{h}} k \Phi(x, y)\left(J_{h}(y) \cdot J_{h}^{\text {test }}(x)-\frac{1}{k^{2}} \operatorname{Div} J_{h}(y) \operatorname{Div} J_{h}^{\text {test }}(x)\right) d s(y) d s(x) \\
=i \int_{\Gamma} E^{i}(x) \cdot J_{h}^{\text {test }}(x) d s(x), \quad \text { for all } J_{h}^{\text {test }}(x) \text { in } V_{h} .
\end{gathered}
$$

where $V_{h}$ is the space spanned by the Rao-Wilson-Glisson basis functions, [9]. The numerical computation amounts to solve a set of linear systems with a full symmetric non hermitian matrix whose size is the number of edges of the mesh. The number of second terms is the number of incident directions times two for the two polarizations. Special attention has been paid in CESC for properly taking into account the singularity of the Green kernel during the assembly process. The LU decomposition of the matrix is performed by means of a set of ScaLAPACK parallel routines. Once the currents have been obtained, the associated far fields are easily deduced (see formula (6.52) page 170 in [3]). To achieve a good precision, the meshing of the scatterers have been done in such a way that the largest lenght of the edges is no more than a tenth of the wavelenght.

Once $\mathcal{F}$ is generated, we corrupt it with random noise. Let $\mathcal{N}$ be a $2 N \times 2 N$ matrix whose entries are random numbers in the interval $[0,1]$ and let $0<\delta<1$ be the noise level. We define the noisy far field matrix $\mathcal{F}_{\delta}$ by

$$
\mathcal{F}_{\delta}:=\mathcal{F}+\delta(\mathcal{N}+i \mathcal{N}) \mathcal{F} .
$$

\subsection{Regularization}

As we mentioned before, we use a Tikhonov regularization to solve the far field equation and use the noisy far field data. This corresponds to solving:

$$
\left(\eta+\mathcal{F}_{\delta}{ }^{*} \mathcal{F}_{\delta}\right) G(z, q)=\mathcal{F}_{\delta}{ }^{*} E_{e, \infty}(z, q),
$$

where $\eta$ is the regularization parameter. If we consider a singular value decomposition of $\mathcal{F}_{\delta}:=U S V^{*}$, then the solution is given by

$$
\left\{V^{*} G(z, q)\right\}_{i}=\frac{S_{i}}{\eta+S_{i}^{2}}\left\{U^{*} E_{e, \infty}(z, q)\right\}_{i}, \quad i=1, \cdots, 2 N .
$$

The regularization parameter is determined using an adapted Morozov's discrepancy principle, [7], by enforcing

$$
\left\|\mathcal{F}_{\delta} G(z, q)-E_{e, \infty}(z, q)\right\|=\left\|\mathcal{F}_{\delta}-\mathcal{F}\right\|\|G(z, q)\| .
$$

INRIA 
Setting $\varepsilon=\left\|\mathcal{F}_{\delta}-\mathcal{F}\right\|$, the regularization parameter is then the non negative solution of

$$
\sum_{i=1}^{2 N} \frac{\eta^{2}-\varepsilon^{2} S_{i}^{2}}{\left(\eta+S_{i}^{2}\right)^{2}}\left|\left\{U^{*} E_{e, \infty}(z, q)\right\}_{i}\right|^{2}=0
$$

\subsection{Numerical procedure}

As it has been pointed out in [1], the reconstruction is sensitive to the the choice of the polarization vector $q$. The numerical experiments have shown that better reconstructions are obtained by combining the values of $G(\cdot, q)$ for three linearly independent vectors $q$.

The following numerical results are obtained by evaluating $\mathcal{G}(z)$ defined by

$$
\mathcal{G}(z)=\frac{1}{3}\left(\left\|G\left(z, q_{1}\right)\right\|^{-1}+\left\|G\left(z, q_{2}\right)\right\|^{-1}+\left\|G\left(z, q_{3}\right)\right\|^{-1}\right)
$$

where $q_{1}=(1,0,0), q_{2}=(0,1,0)$ and $q_{3}=(0,0,1)$.

We consider a uniform mesh of the region that contains the scatterer and evaluate $\mathcal{G}$ for every vertex $z_{i}$ of this mesh. The 3 -D reconstruction is obtained by plotting the surface

$$
G(z)=C \max _{z_{i}} G\left(z_{i}\right) .
$$

We then vary the value of $C$ till obtaining the "best visual" reconstruction.

\section{$5 \quad$ Numerical results}

All the following numerical results correspond to perfect scatterers $z_{r}=0$ and to an added noise level $\delta=1 \%$.

\subsection{The case of the sphere}

We shall here numerically validate the analytical results of section 3 . We consider a sphere of radius 1 and plot the isovalues of the $G(z)$ in the plane $z_{3}=0$. Figures 13 and 14 correspond to a frequency $k=3$ and a number of directions $N=42$. Figures 15 and 16 correspond to a frequency $k=6$ and a number of directions $N=162$. Figures 17 and 18 correspond to a frequency $k=9$ and a number of directions $N=492$. We observe that the influence of the frequency on the accuracy of the result is more clear in the case of LSM than in the case of the factorization method (FM). As observed in the Al analytical study, when one increases the frequency, the transition region between small and large values is reduced and becomes closer to the true scatterer surface.

Qualitatively, the LSM seems to provide better 3-D reconstructions than the factorization method (FM). 


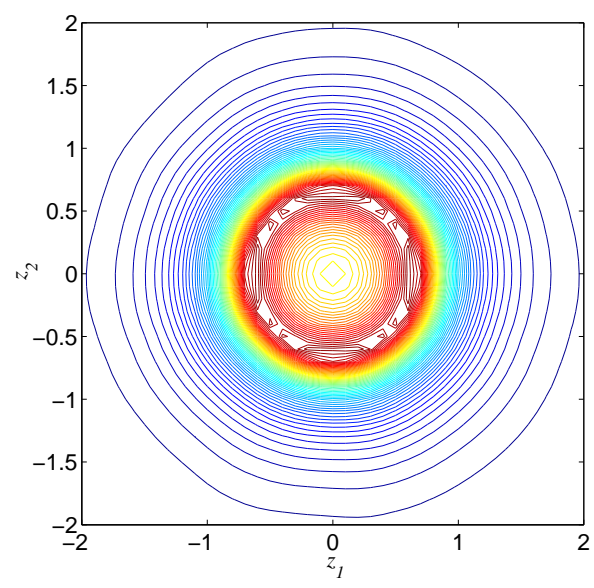

Figure 13: LSM: Isolines of $\mathcal{G}$ for $k=3$.

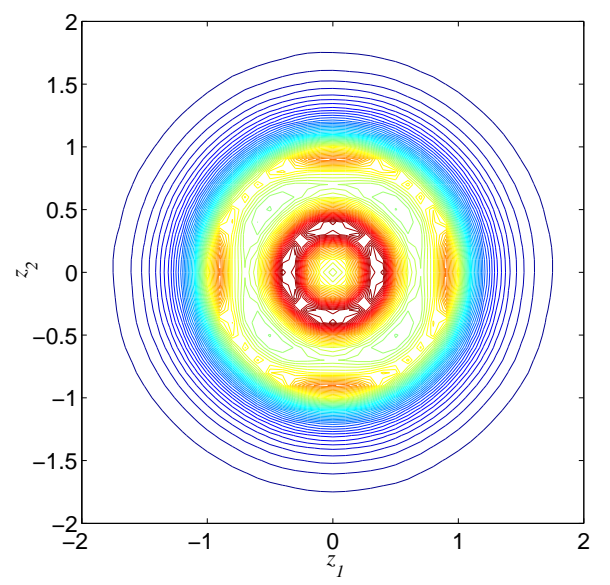

Figure 15: LSM: Isolines of $\mathcal{G}$ for $k=6$.

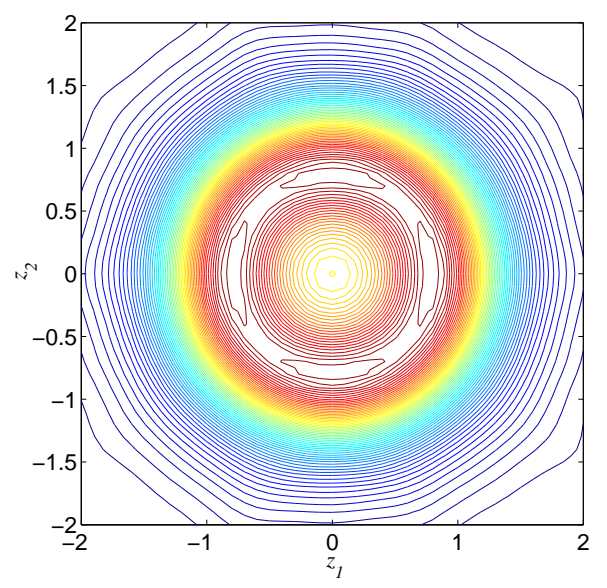

Figure 14: FM: Isolines of $\mathcal{G}$ for $k=3$.

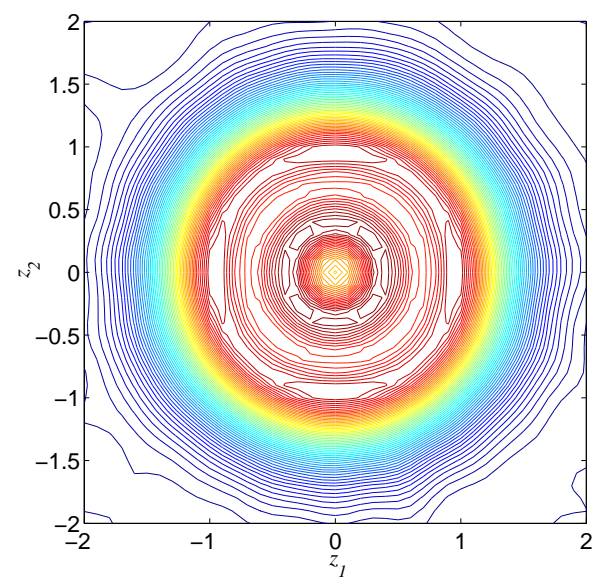

Figure 16: FM: Isolines of $\mathcal{G}$ for $k=6$. 


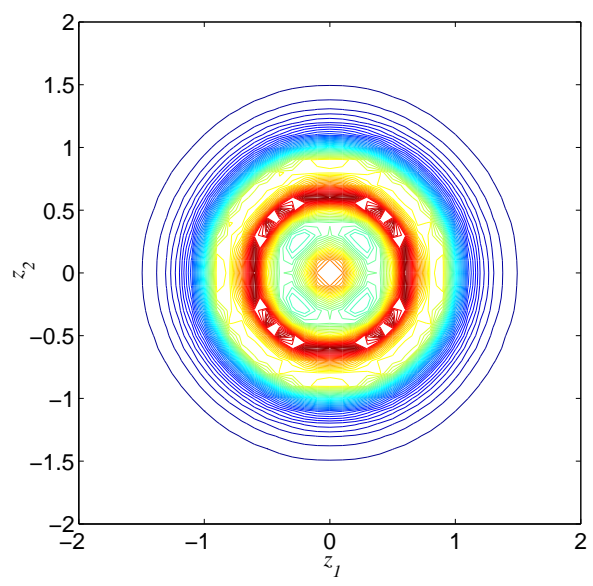

Figure 17: LSM: Isolines of $\mathcal{G}$ for $k=9$.

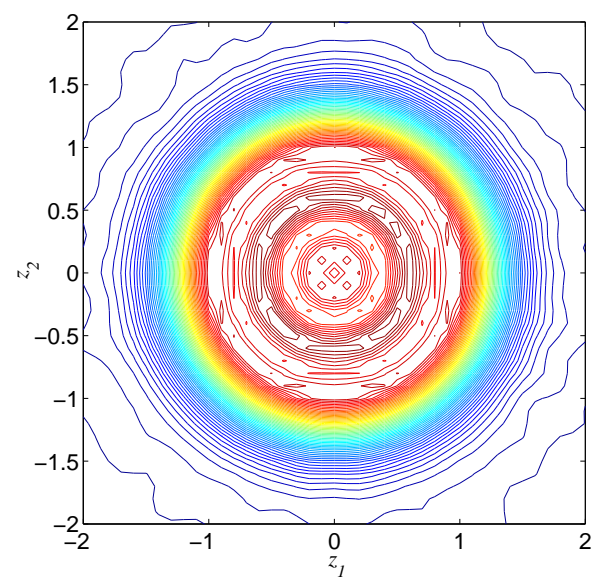

Figure 18: FM: Isolines of $\mathcal{G}$ for $k=9$.

\subsection{The analysis of multiple reflections}

This section is concerned with the reconstruction of two disconnected objects in the cases where multiple reflections between the two objects are significant. The geometry of our example is given in Figure 19.

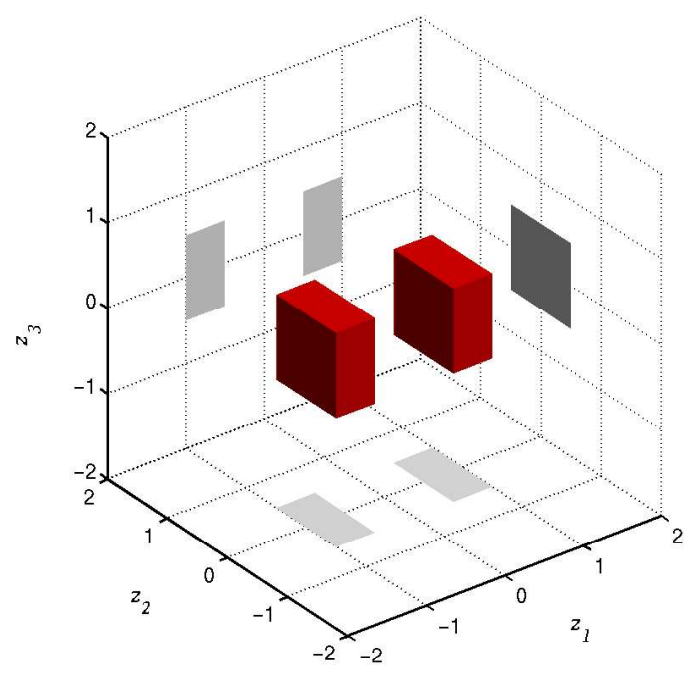

Figure 19: Exact geometry. 
The following reconstructions are done using data on a full aperture. First we use a frequency $k=3$ that corresponds to wavelength twice as long as the distance between the two objects. Using $2 \times 42$ incident waves, the reconstructed object is in Figure 20 (the wavelength is indicated by a thick line on the axis $z_{1}$ ).

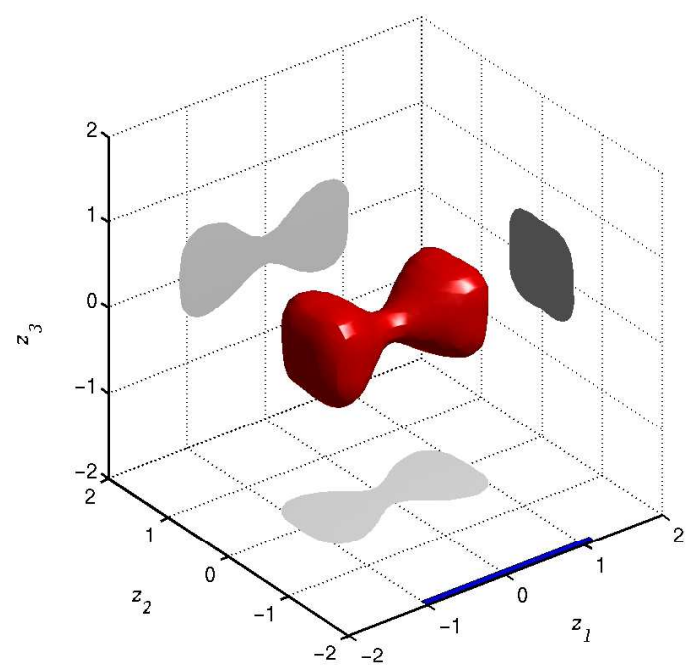

Figure 20: Reconstructed geometry.

One observe that the distinction between the presence of two objects or a single one is not possible. This is also confirmed by the isolines of $\mathcal{G}$ presented in Figure 21.
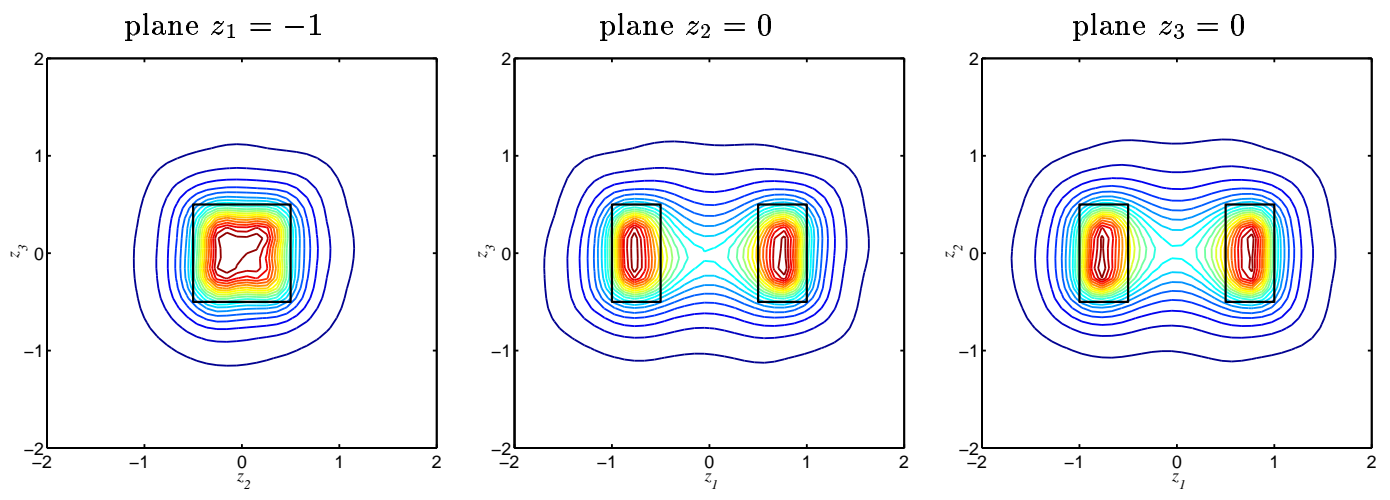

Figure 21: 20 isolines of $\mathcal{G}$ for different cross sections. $\mathrm{k}=3$.

Then we increase the frequency and use $k=6$ (and use $2 \times 162$ incident plane waves). Figure 22 shows the reconstructed objects where one can now distinguish 2 distinct objects.

INRIA 
This improvement is only due to the use of a higher frequency and is not due to the larger number of used incident waves. For instance one obtains the same reconstruction as the one in Figure 20 if we use $k=3$ and $2 \times 162$ incident plane waves.

Increasing the frequency has also sharpened the corners in the $3-\mathrm{D}$ reconstruction.

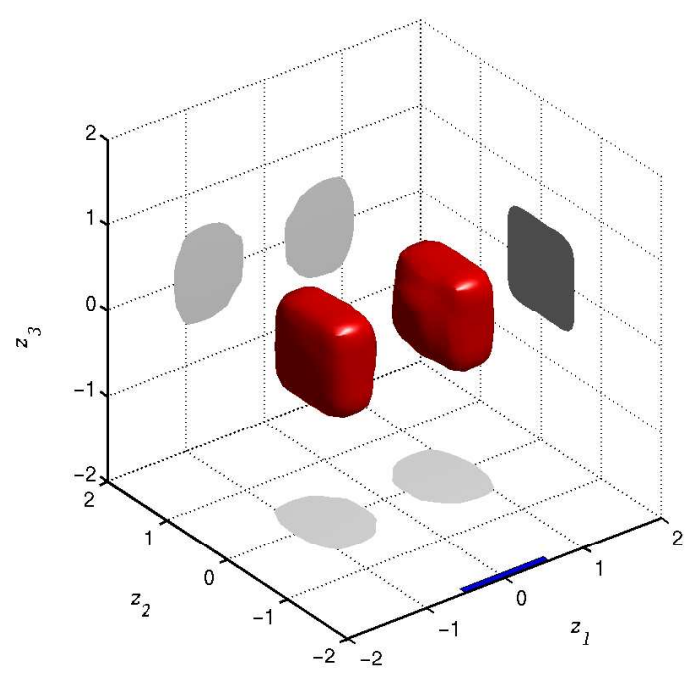

Figure 22: Reconstructed geometry.

These observations are also confirmed by the isolines of $\mathcal{G}$ presented in Figure 23 .
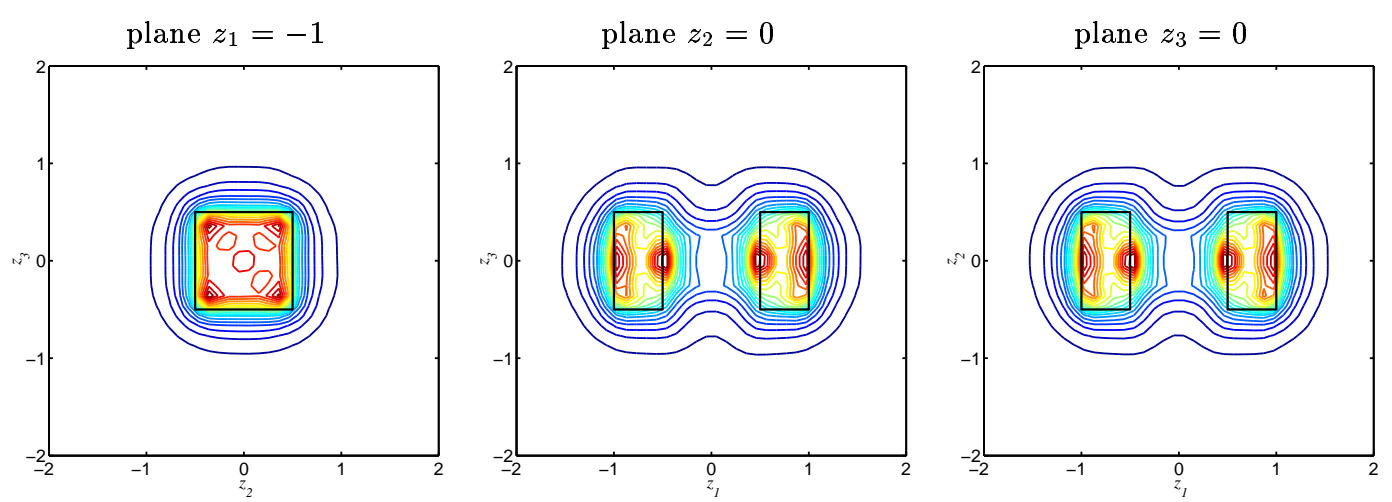

Figure 23: 20 isolines of $\mathcal{G}$ for different cross sections. $\mathrm{k}=6$. 


\subsection{Reconstruction of complex geometries}

We are concerned in this section with the reconstruction of complex geometries, in the sense that they contain small details and are not smooth. We choose two examples. The first one corresponds to a teapot presented in Figure 24 and the second one corresponds to an aircraft presented in Figure 29.

\subsubsection{Reconstruction of a teapot}

We consider here the case of full aperture experiments. The exact geometry is presented in Figure 24 .

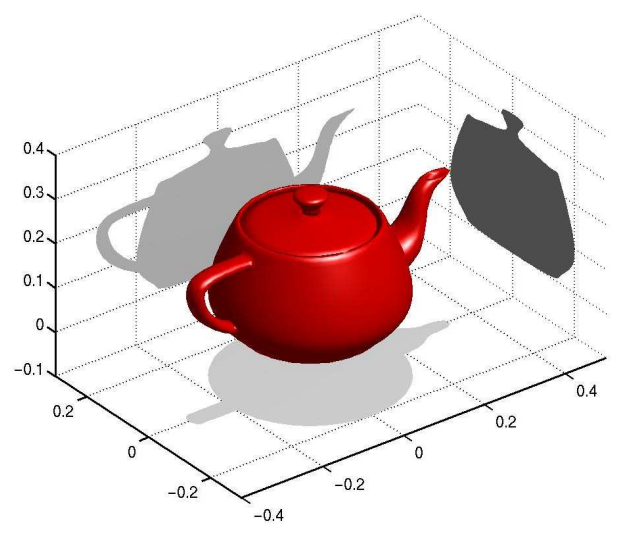

Figure 24: Exact geometry of the teapot.

Figures 25, 26, 27 and 28 show the 3 -D reconstructions using respectively $(k, N)=$ $(28,252),(k, N)=(56,252),(k, N)=(84,492)$ and $(96,492)$ where $k$ denotes the frequency and where $N$ denotes the number of vertices used in the unit sphere mesh (see section 4.1).

One clearly observe how the increase of the frequency helps a better resolution of the reconstruction. The last figure shows non-smoothness in the reconstruction that may be due to the lack of accuracy in the synthetic data. For this high frequency experiment we did the forward computations of the scattered fields using 6 points per wavelength only! 


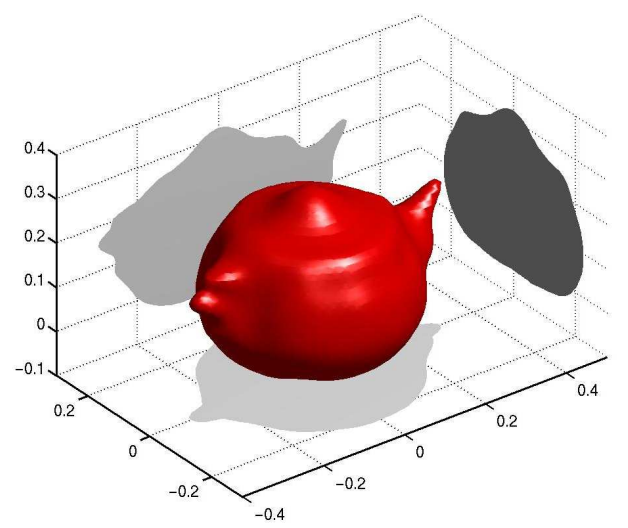

Figure 25: Reconstruction of the teapot with $\mathrm{k}=28$, using 252 incident directions.

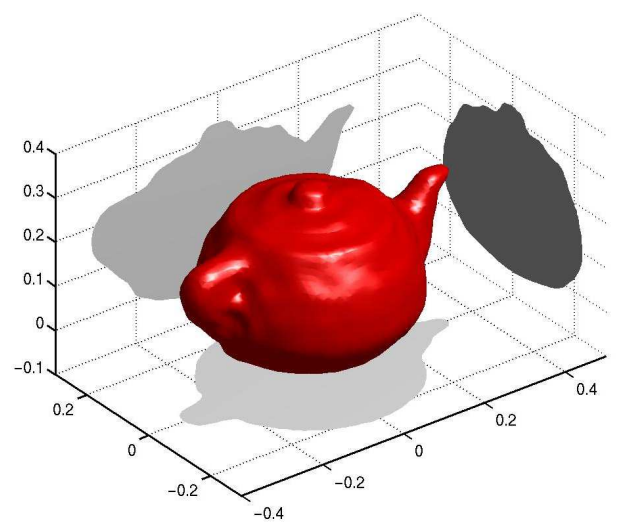

Figure 26: Reconstruction of the teapot with $\mathrm{k}=56$, using 252 incident directions. 


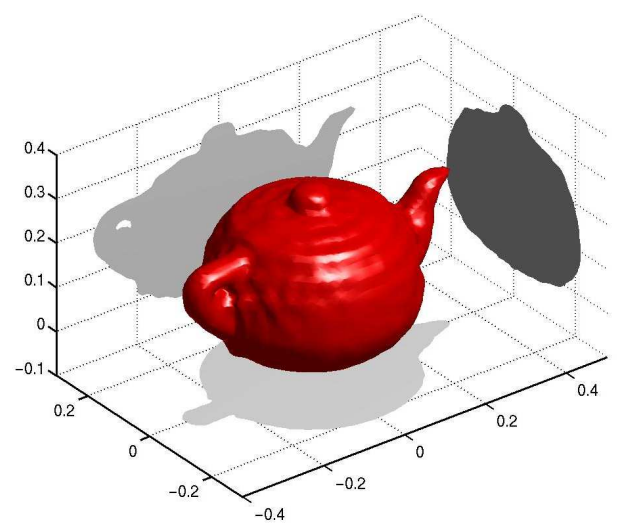

Figure 27: Reconstruction of the teapot with $\mathrm{k}=84$, using 492 incident directions.

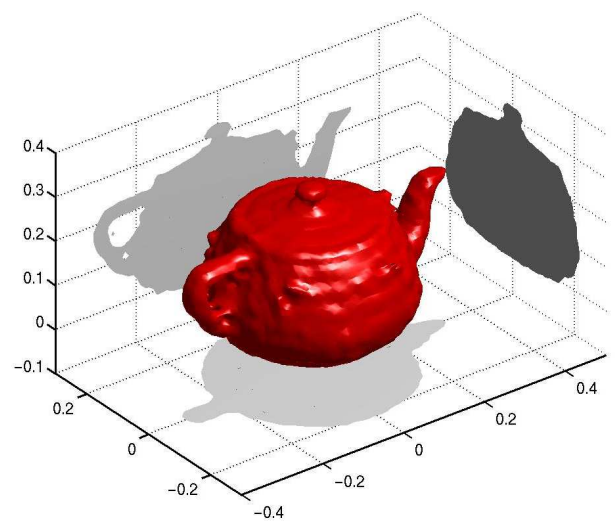

Figure 28: Reconstruction of the teapot with $\mathrm{k}=96$, using 492 incident directions. 


\subsubsection{Reconstruction of an aircraft}

This is another example of a (complex geometry, see Fig. 29) reconstruction using a full aperture data (for instance 252 uniform directions are used (Fig. 30)). In the first case $k=2 \pi$ (the wavelength is twice the diameter of the aircraft), one obtains a low resolution reconstruction, see Fig. 31. For instance, one cannot distinguish small details such as engines, but larger parts such as wings are visible. In the second case (see Fig. 32) the frequency is doubled $(k=4 \pi)$ and one can observe how the reconstruction resolution is highly improved.

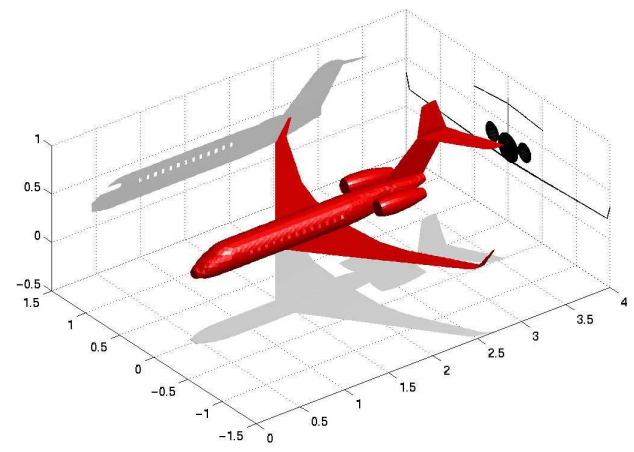

Figure 29: The aircraft exact geometry

\subsection{Limited aperture experiments}

We retain here the example of an aircraft (see Figure 29) and the frequency $k=4 \pi$ for the reconstruction. However we restrict the set of observation points to only one quarter of the unit sphere. Figures 34 and 36 display the reconstruction results that respectively correspond to the lower left quarter (Fig. 33) and the lower right quarter (Fig. 35). We observe in both cases that the reconstruction is less spectacular than in the case of full aperture, however we still distinguish the main features of the aircraft. Comparing the two cases we also observe that the illuminated region of the aircraft corresponds to the best reconstructed part. 


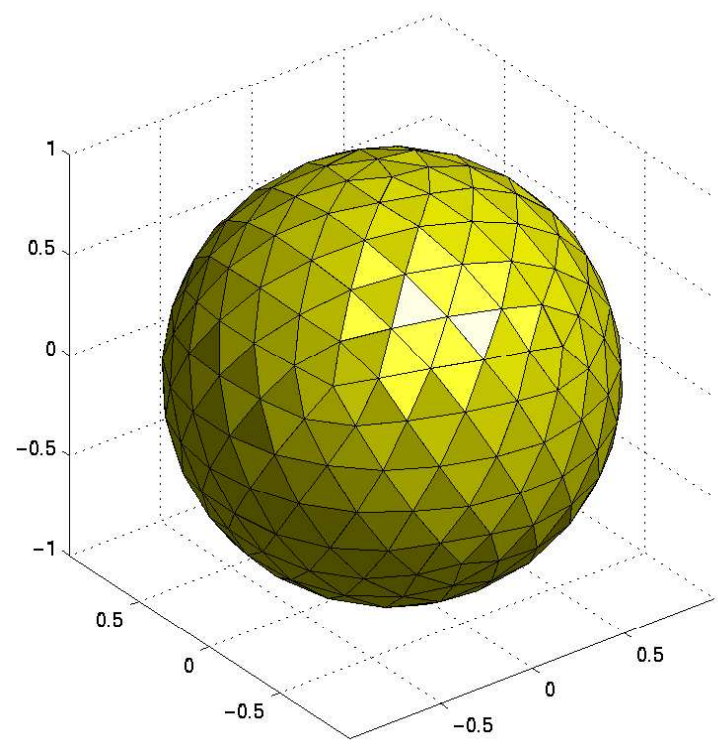

Figure 30: Mesh of the full aperture: 252 vertices

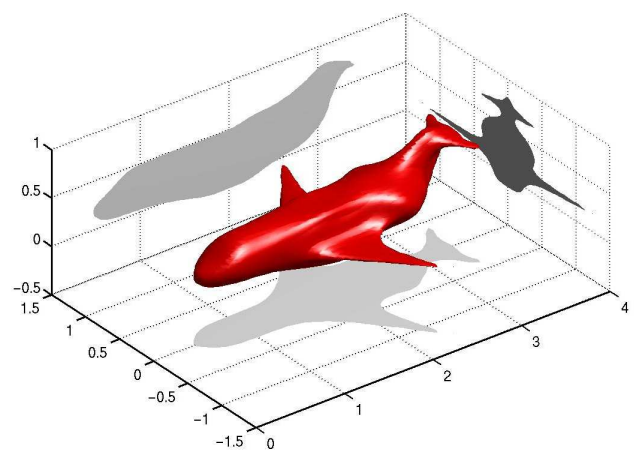

Figure 31: Reconstructed aircraft: $k=2 \pi$ 


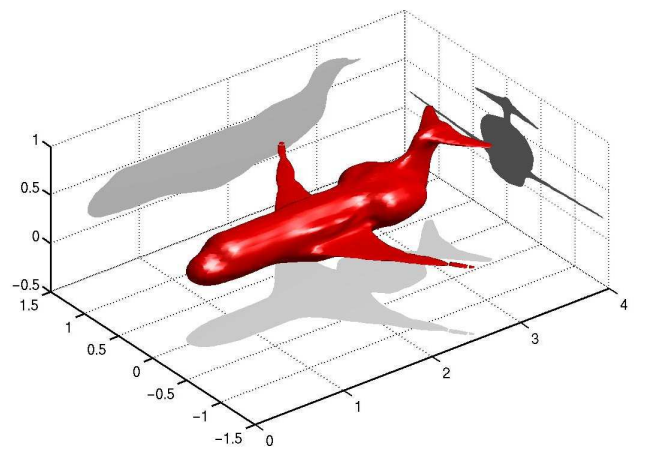

Figure 32: Reconstructed aircraft: $k=4 \pi$

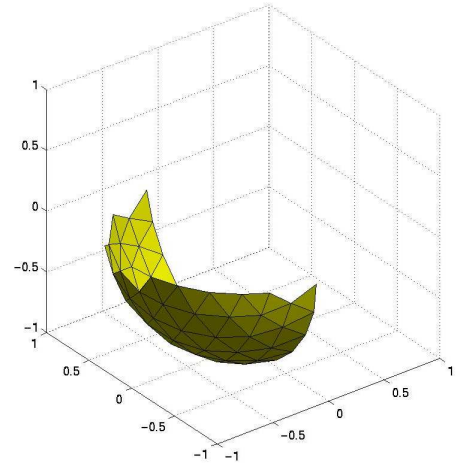

Figure 33: Mesh of $\Gamma$ (64 vertices)

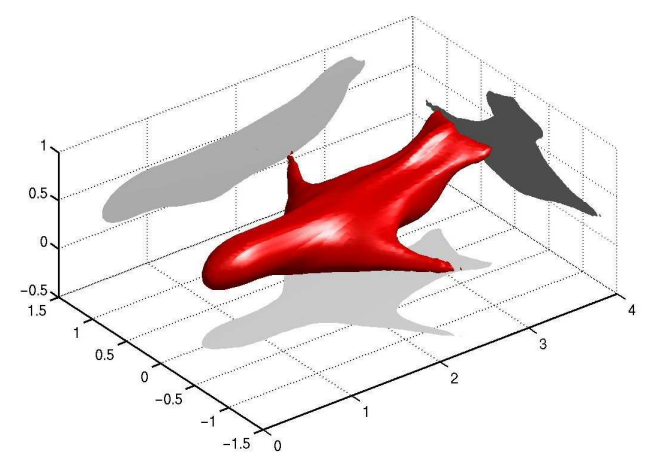

Figure 34: Reconstructed aircraft $(k=4 \pi)$

$\mathrm{RR} \mathrm{n}^{\circ} 4665$ 


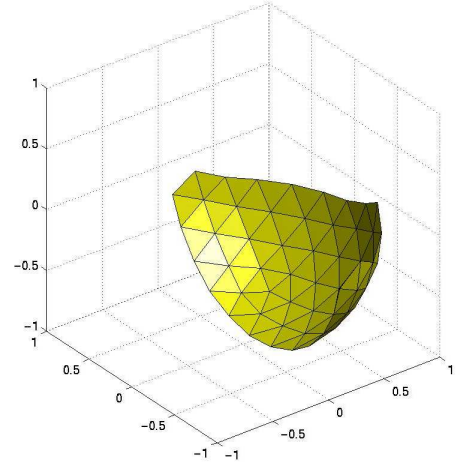

Figure 35: Mesh of $\Gamma$ (61 vertices)

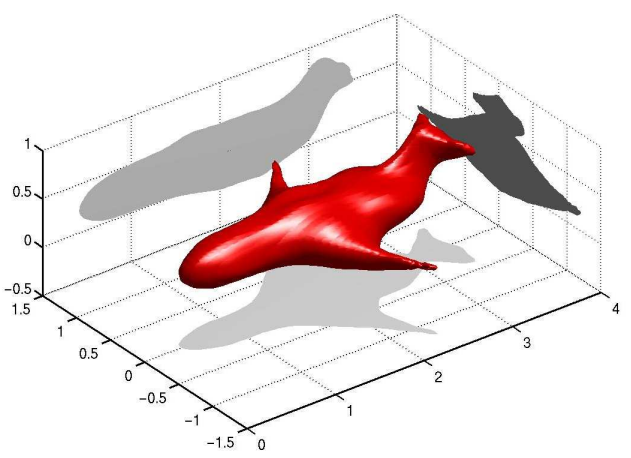

Figure 36: Reconstructed aircraft $(k=4 \pi)$

\section{A Appendix}

\section{A.1 Decomposition of the far field of a magnetic dipole}

We recall the definitions of section 3 . We first introduce the functions

$$
u_{n}^{m}(x)=j_{n}(k|x|) Y_{n}^{m}(\hat{x}), \quad v_{n}^{m}(x)=h_{n}^{(1)}(k|x|) Y_{n}^{m}(\hat{x}), \quad x=|x| \hat{x},
$$

where $\left(Y_{n}^{m}\right)$ denotes the set of orthonormal spherical harmonics, $\left(j_{n}\right)$ denotes the set of spherical Bessel functions and $\left(h_{n}^{(1)}\right)$ denotes the set of spherical Hankel functions of the first kind. We then define the sets $\left(M_{n}^{m}\right)$ and $\left(N_{n}^{m}\right)$ of respectively entire and radiating solutions to Maxwell's equations by:

$$
M_{n}^{m}(x)=\operatorname{curl}\left(x u_{n}^{m}(x)\right), \quad N_{n}^{m}(x)=\operatorname{curl}\left(x v_{n}^{m}(x)\right) .
$$

We also define the vector spherical harmonics of order $n$

$$
U_{n}^{m}(\hat{x})=\frac{1}{\sqrt{n(n+1)}} \operatorname{Grad} Y_{n}^{m}(\hat{x}), \quad V_{n}^{m}(\hat{x})=\frac{1}{\sqrt{n(n+1)}} \hat{x} \times \operatorname{Grad} Y_{n}^{m}(\hat{x})
$$

where $\hat{x}$ is a point on the unit sphere $\Omega$. We recall that the set of vector spherical harmonics form an orthonormal basis of $T^{2}(\Omega)$.

Lemma A.1 Let $q$ be some given vector in $\mathbb{R}^{3}, z$ be some given point in $\mathbb{R}^{3}$ and $\hat{x}$ be some direction on $\Omega$. The far field associated to the magnetic dipole located at point $z$ and with momentum $q$, defined by

$$
E^{\infty}(\hat{x} ; z, q):=-\frac{i k}{4 \pi}(q \times \hat{x})
$$


has the following expansion

$$
\begin{aligned}
E^{\infty}(\hat{x} ; z, q)= & \sum_{n=1}^{\infty} \frac{(-i)^{n}}{\sqrt{n(n+1)}} \sum_{m=-n}^{n}\left(i k \overline{M_{n}^{m}}(z) \cdot q\right) U_{n}^{m}(\hat{x}) \\
& +\sum_{n=1}^{\infty} \frac{(-i)^{n}}{\sqrt{n(n+1)}} \sum_{m=-n}^{n}\left(\operatorname{curl} \overline{M_{n}^{m}}(z) \cdot q\right) V_{n}^{m}(\hat{x})
\end{aligned}
$$

Proof. Let $q \in \mathbb{R}^{3}$ and let $x$ and $z$ be two points of $\mathbb{R}^{3}$ such that $|x|>|z|$. If $\Phi(x, z)$ denotes the Green function for the Helmholtz equation, then ([3], Theorem 6.7 page 178)

$$
\begin{aligned}
\Phi(x, z) q= & i k \sum_{m=-n}^{n} \frac{1}{n(n+1)} \sum_{n=1}^{\infty} N_{n}^{m}(x) \overline{M_{n}^{m}}(z) \cdot q \\
& +\frac{i}{k} \sum_{n=1}^{\infty} \frac{1}{n(n+1)} \sum_{m=-n}^{n} \operatorname{curl} N_{n}^{m}(x) \operatorname{curl} \overline{M_{n}^{m}}(z) \cdot q \\
& +\frac{i}{k} \sum_{n=1}^{\infty} \frac{1}{n(n+1)} \sum_{m=-n}^{n} \operatorname{grad} v_{n}^{m}(x) \operatorname{grad} \overline{u_{n}^{m}}(z) \cdot q,
\end{aligned}
$$

We take the curl of the two terms of this relationship and use

$$
\operatorname{curl} \operatorname{curl} N_{n}^{m}(x)=k^{2} N_{n}^{m}(x),
$$

we get

$$
\begin{aligned}
\operatorname{curl} \Phi(x, z) q= & i k \sum_{m=-n}^{n} \frac{1}{n(n+1)} \sum_{n=1}^{\infty} \operatorname{curl} N_{n}^{m}(x) \overline{M_{n}^{m}}(z) \cdot q \\
& +i k \sum_{n=1}^{\infty} \frac{1}{n(n+1)} \sum_{m=-n}^{n} \operatorname{curl} \operatorname{curl} N_{n}^{m}(x) \operatorname{curl} \overline{M_{n}^{m}}(z) \cdot q .
\end{aligned}
$$

According to Theorem 6.26 page 175 of [3], if

$$
E(x)=\sum_{n=1}^{\infty} \sum_{m=-n}^{n} \xi_{n}^{m} N_{n}^{m}(x)+\chi_{n}^{m} \operatorname{curl} N_{n}^{m}(x),
$$

then

$$
E(x) \simeq E^{\infty}(\hat{x}) \frac{e^{i k|x|}}{|x|}\left(1+O\left(\frac{1}{|x|}\right)\right)
$$

with

$$
E^{\infty}(\hat{x})=\frac{1}{i k} \sum_{n=1}^{\infty}(-i)^{n} \sum_{m=-n}^{n}\left(i k \chi_{n}^{m} \operatorname{Grad} Y_{n}^{m}(\hat{x})-\xi_{n}^{m} \hat{x} \times \operatorname{Grad} Y_{n}^{m}(\hat{x})\right)
$$


In our case, we have

$$
\xi_{n}^{m}=\frac{i k}{n(n+1)} \operatorname{curl} \overline{M_{n}^{m}}(z) \cdot q, \quad \chi_{n}^{m}=\frac{i k}{n(n+1)} \overline{M_{n}^{m}}(z) \cdot q,
$$

and the far field associated to the magnetic dipole located at point $z$ and with momentum $q$ is

$$
\begin{aligned}
E^{\infty}(\hat{x} ; z, q)= & \sum_{n=1}^{\infty} \frac{(-i)^{n}}{n(n+1)} \sum_{m=-n}^{n}\left(i k \overline{M_{n}^{m}}(z) \cdot q\right) \operatorname{Grad}_{n}^{m}(\hat{x}) \\
& +\sum_{n=1}^{\infty} \frac{(-i)^{n}}{n(n+1)} \sum_{m=-n}^{n}\left(\operatorname{curl} \overline{M_{n}^{m}}(z) \cdot q\right)\left(\hat{x} \times \operatorname{Grad}_{n}^{m}(\hat{x})\right) .
\end{aligned}
$$

Otherwise, we know the explicit form of the far field associated to a magnetic dipole, whence, if $p$ is some vector

$$
\left\{\begin{array}{c}
-\frac{i k}{4 \pi}(q \times \hat{x}) \cdot p e^{-i k z \hat{x}}= \\
\sum_{n=1}^{\infty} \frac{(-i)^{n}}{n(n+1)} \sum_{m=-n}^{n}\left(i k \overline{M_{n}^{m}}(z) \cdot q\right) \operatorname{Grad} Y_{n}^{m}(\hat{x}) \cdot p \\
+\sum_{n=1}^{\infty} \frac{(-i)^{n}}{n(n+1)} \sum_{m=-n}^{n}\left(\operatorname{curl} \overline{M_{n}^{m}}(z) \cdot q\right)\left(\hat{x} \times \operatorname{Grad} Y_{n}^{m}(\hat{x})\right) \cdot p
\end{array}\right.
$$

This formula will also be useful in the following. Finally, using the orthonormal basic functions $U_{n}^{m}$ and $V_{n}^{m}$, we get (35).

\section{A.2 Determination of the scattered field by a sphere}

\section{A.2.1 The problem}

We consider the scattering of an electromagnetic incident plane wave $\left(E^{i}, H^{i}\right)$ by a sphere of radius $a$. The scattered electromagnetic field $\left(E^{s}, H^{s}\right)$ is assumed to satisfy to the boundary condition

$$
\hat{x} \times\left(\left(E^{i}(a \hat{x})+E^{s}(a \hat{x})\right) \times \hat{x}\right)+z_{r} \hat{x} \times\left(H^{i}(a \hat{x})+H^{s}(a \hat{x})\right)=0, \forall \hat{x},|\hat{x}|=1 .
$$

where $z_{r}$ is some complex number with $\Re e\left(z_{r}\right) \leq 0$. The aim of what follows is to give a closed expression of the far field associated to the pair $\left(E_{s}, H_{s}\right)$. The calculations are classical (see for instance [11], page 259 for the scattering of a dielectric sphere by an incident electromagnetic field of momentum $\hat{d}=\hat{z}$ and momentum $q=\hat{x}$ ). The process can be divided into four steps; First, the incident plane wave is expressed in terms of Debye 
potentials, i.e. a is decomposed according to

$$
\begin{aligned}
& E^{i}(x ; \hat{d}, p)=\operatorname{curl}\left(x v^{i}(x ; \hat{d}, p)\right)+\frac{i}{k} \operatorname{curl} \operatorname{curl}\left(x u^{i}(x ; \hat{d}, p)\right) \\
& H^{i}(x ; \hat{d}, p)=-\frac{i}{k} \operatorname{curl} \operatorname{curl}\left(x v^{i}(x ; \hat{d}, p)\right)+\operatorname{curl}\left(x u^{i}(x ; \hat{d}, p)\right),
\end{aligned}
$$

where the two potentials $u^{i}(x ; \hat{d}, p)$ and $v^{i}(x ; \hat{d}, p)$ are some solutions of the Helmholtz equation. Second, the scattered field is sought in an analogous form, i.e.

$$
\begin{aligned}
& E^{s}(x ; \hat{d}, p)=\operatorname{curl}\left(x v^{s}(x ; \hat{d}, p)\right)+\frac{i}{k} \operatorname{curl} \operatorname{curl}\left(x u^{s}(x ; \hat{d}, p)\right) \\
& H^{s}(x ; \hat{d}, p)=-\frac{i}{k} \operatorname{curl} \operatorname{curl}\left(x v^{s}(x ; \hat{d}, p)\right)+\operatorname{curl}\left(x u^{s}(x ; \hat{d}, p)\right),
\end{aligned}
$$

where the two potentials $u^{s}(x ; \hat{d}, p)$ and $v^{s}(x ; \hat{d}, p)$ are some radiating solutions of the Helmholtz equation. Third, the boundary condition is used to get the expression of the scattered Debye Potential. Finally, the far field is obtained using Theorem 6.26 page 175 of [3].

\section{A.2.2 Decomposition of a plane wave in term of Debye potentials}

Lemma A.2 Let $p$ be some polarization vector, $\hat{d}$ be some incident direction $(\hat{d} \mid=1)$ and $k$ be some wavenumber. We have

$$
\left\{\begin{aligned}
E^{i}(x ; \hat{d}, p) & :=i k(\hat{d} \times p) \times \hat{d} e^{i k x \hat{d}} \\
& =\operatorname{curl}\left(x v^{i}(x ; \hat{d}, p)\right)+\frac{i}{k} \operatorname{curl} \operatorname{curl}\left(x u^{i}(x ; \hat{d}, p)\right) \\
H^{i}(x ; \hat{d}, p) & :=i k \hat{d} \times p e^{i k x \hat{d}} \\
& =-\frac{i}{k} \operatorname{curl} \operatorname{curl}\left(x v^{i}(x ; \hat{d}, p)\right)+\operatorname{curl}\left(x u^{i}(x ; \hat{d}, p)\right)
\end{aligned}\right.
$$

with

$$
\begin{aligned}
& u^{i}(x ; \hat{d}, p)=\sum_{n=1}^{\infty} \sum_{m=-n}^{n} a_{n}^{m}(\hat{d}, p) j_{n}(k|x|) Y_{n}^{m}(\hat{x}) \\
& v^{i}(x ; \hat{d}, p)=\sum_{n=1}^{\infty} \sum_{m=-n}^{n} b_{n}^{m}(\hat{d}, p) j_{n}(k|x|) Y_{n}^{m}(\hat{x}) .
\end{aligned}
$$


and

$$
\begin{aligned}
& a_{n}^{m}(\hat{d}, p)=-i^{n} \frac{4 \pi i k}{n(n+1)} \operatorname{Grad} \overline{Y_{n}^{m}}(\hat{d}) \cdot p \\
& b_{n}^{m}(\hat{d}, p)=-i^{n} \frac{4 \pi i k}{n(n+1)}\left(\hat{d} \times \operatorname{Grad}_{n}^{m}(\hat{d})\right) \cdot p,
\end{aligned}
$$

Proof. An elegant way to get this decomposition is to start from (36): we set $\hat{x}=\hat{d}$, $z=x$ and after complex conjugation, we obtain

$$
\left\{\begin{array}{c}
\frac{i k}{4 \pi}(q \times \hat{d}) \cdot p e^{i k x \hat{d}}=\frac{i k}{4 \pi}(\hat{d} \times p) e^{i k x \hat{d}} \cdot q= \\
\sum_{n=1}^{\infty} \frac{i^{n}}{n(n+1)} \sum_{m=-n}^{n}\left(-i k M_{n}^{m}(x) \cdot q\right) \operatorname{Grad} \overline{Y_{n}^{m}}(\hat{d}) \cdot p \\
+\sum_{n=1}^{\infty} \frac{i^{n}}{n(n+1)} \sum_{m=-n}^{n}\left(\operatorname{curl} M_{n}^{m}(x) \cdot q\right)\left(\hat{d} \times \operatorname{Grad} \overline{Y_{n}^{m}}(\hat{d})\right) \cdot p
\end{array}\right.
$$

or, using the explicit form of $M_{n}^{m}(x)$,

$$
\begin{gathered}
H^{i}(x ; \hat{d}, p) \cdot q= \\
\operatorname{curl}\left(x \sum_{n=1}^{\infty} \frac{4 \pi i^{n}}{n(n+1)} \sum_{m=-n}^{n}\left(-i k j_{n}(k|x|) Y_{n}^{m}(\hat{x})\right) \operatorname{Grad} \overline{Y_{n}^{m}}(\hat{d}) \cdot p\right) \cdot q \\
-\frac{1}{i k} \operatorname{curlcurl}\left(x \sum_{n=1}^{\infty} \frac{4 \pi i^{n}}{n(n+1)}\right. \\
\left.\sum_{m=-n}^{n}\left(-i k j_{n}(k|x|) Y_{n}^{m}(\hat{x})\right)\left(\hat{d} \times \operatorname{Grad} \overline{Y_{n}^{m}}(\hat{d})\right) \cdot p\right) \cdot q
\end{gathered}
$$

the potentials are finally

$$
\begin{aligned}
& u^{i}(x ; \hat{d}, p)=-\sum_{n=1}^{\infty} \frac{4 i \pi k i^{n}}{n(n+1)} \sum_{m=-n}^{n}\left(\operatorname{Grad} \overline{Y_{n}^{m}}(\hat{d}) \cdot p\right) j_{n}(k|x|) Y_{n}^{m}(\hat{x}) \\
& v^{i}(x ; \hat{d}, p)=-\sum_{n=1}^{\infty} \frac{4 i \pi k i^{n}}{n(n+1)} \sum_{m=-n}^{n}\left(\left(\hat{d} \times \operatorname{Grad} \overline{Y_{n}^{m}}(\hat{d})\right) \cdot p\right) j_{n}(k|x|) Y_{n}^{m}(\hat{x}),
\end{aligned}
$$

and the result follows.

INRIA 


\section{A.2.3 Determination of the scattered field}

We seek the scattered field in the form

$$
\begin{aligned}
& E^{s}(x ; \hat{d}, p)=\operatorname{curl}\left(x v^{s}(x ; \hat{d}, p)\right)+\frac{i}{k} \operatorname{curl} \operatorname{curl}\left(x u^{s}(x ; \hat{d}, p)\right) \\
& H^{s}(x ; \hat{d}, p)=-\frac{i}{k} \operatorname{curl} \operatorname{curl}\left(x v^{s}(x ; \hat{d}, p)\right)+\operatorname{curl}\left(x u^{s}(x ; \hat{d}, p)\right),
\end{aligned}
$$

with

$$
\begin{aligned}
& u^{s}(x ; \hat{d}, p)=\sum_{n=1}^{\infty} \sum_{m=-n}^{n} u_{n} a_{n}^{m}(\hat{d}, p) h_{n}^{(1)}(k|x|) Y_{n}^{m}(\hat{x}) \\
& v^{s}(x ; \hat{d}, p)=\sum_{n=1}^{\infty} \sum_{m=-n}^{n} v_{n} b_{n}^{m}(\hat{d}, p) h_{n}^{(1)}(k|x|) Y_{n}^{m}(\hat{x}),
\end{aligned}
$$

(we have simply replaced the $j_{n}$ 's by the $h_{n}^{(1)}$ 's (to ensure a radiating field) and have introduced some unknown coefficients in front of each term of the series). It can be readily verified that $\left(E_{s}, H_{s}\right)$ is a radiating solution of the Maxwell's equation for all coefficients $\left(u_{n}, v_{n}\right)$ bounded in $n$. The unknown coefficients are determined by the boundary condition (37). Their expressions involve the Ricatti Bessel functions whose definition is

$$
\psi_{n}(t)=t j_{n}(t), \quad \zeta_{n}^{(1)}(t)=t h_{n}^{(1)}(t)=t\left(j_{n}(t)+i y_{n}(t) .\right.
$$

Lemma A.3 Let $\left(E_{i}, H_{i}\right)$ and $\left(E_{s}, H_{s}\right)$ be respectively defined by (38)-(39) and (41)-(42), through (40). The boundary condition

$$
\hat{x} \times\left(\left(E^{i}+E^{s}\right) \times \hat{x}\right)+z_{r} \hat{x} \times\left(H^{i}+H^{s}\right)=0,
$$

is satisfied on the surface $|x|=a$ for

$$
\begin{aligned}
& u_{n}=u_{n}\left(k a, z_{r}\right)=-\frac{i \psi_{n}{ }^{\prime}(k a)+z_{r} \psi_{n}(k a)}{i \zeta_{n}^{(1)^{\prime}}(k a)+z_{r} \zeta_{n}^{(1)}(k a)} \\
& v_{n}=v_{n}\left(k a, z_{r}\right)=-\frac{\psi_{n}(k a)+i z_{r} \psi_{n}{ }^{\prime}(k a)}{\zeta_{n}^{(1)}(k a)+i z_{r} \zeta_{n}^{(1)^{\prime}}(k a)} .
\end{aligned}
$$

Proof. The total electromagnetic field $(E, H)=\left(E^{i}+E^{s}, H^{i}+H^{s}\right)$ reads

$$
\begin{aligned}
& E(x)=\sum_{n=1}^{\infty} \sum_{m=-n}^{n} b_{n}^{m}\left(M_{n}^{m}(x)+v_{n} N_{n}^{m}(x)\right)+\frac{i}{k} a_{n}^{m} \operatorname{curl}\left(M_{n}^{m}(x)+u_{n} N_{n}^{m}(x)\right), \\
& H(x)=\sum_{n=1}^{\infty} \sum_{m=-n}^{n} a_{n}^{m}\left(M_{n}^{m}(x)+u_{n} N_{n}^{m}(x)\right)-\frac{i}{k} b_{n}^{m} \operatorname{curl}\left(M_{n}^{m}(x)+v_{n} N_{n}^{m}(x)\right) .
\end{aligned}
$$

$\mathrm{RR} \mathrm{n}^{\circ} 4665$ 
where

$$
M_{n}^{m}(x)=\operatorname{curl}\left(x j_{n}(k|x|) Y_{n}^{m}(\hat{x})\right), \quad N_{n}^{m}(x)=\operatorname{curl}\left(x h_{n}^{(1)}(k|x|) Y_{n}^{m}(\hat{x})\right) .
$$

Using formula (6.64) and (6.65) of [3] page 173, we have

$$
\left(M_{n}^{m}(x)+v_{n} N_{n}^{m}(x)\right)=-\frac{1}{k|x|}\left(\psi_{n}(k|x|)+v_{n} \zeta_{n}^{(1)}(k|x|)\right)\left(\hat{x} \times \operatorname{Grad} Y_{n}^{m}(\hat{x})\right),
$$

and

$$
\begin{gathered}
\hat{x} \times\left(\operatorname{curl}\left(M_{n}^{m}(x)+v_{n} N_{n}^{m}(x)\right) \times \hat{x}\right)= \\
\frac{1}{|x|}\left(\psi_{n}{ }^{\prime}(k|x|)+v_{n} \zeta_{n}^{(1)^{\prime}}(k|x|)\right) \operatorname{Grad} Y_{n}^{m}(\hat{x}) .
\end{gathered}
$$

From this, we obtain

$$
\begin{gathered}
\hat{x} \times(E(x) \times \hat{x})= \\
\frac{1}{k|x|} \sum_{n=1}^{\infty} \sum_{m=-n}^{n}\left(-b_{n}^{m}\right)\left(\psi_{n}(k|x|)+v_{n} \zeta_{n}^{(1)}(k|x|)\right)\left(\hat{x} \times \operatorname{Grad}_{n}^{m}(\hat{x})\right) \\
+\frac{1}{k|x|} \sum_{n=1}^{\infty} \sum_{m=-n}^{n} i a_{n}^{m}\left(\psi_{n}{ }^{\prime}(k|x|)+u_{n} \zeta_{n}^{(1)^{\prime}}(k|x|)\right) \operatorname{Grad} Y_{n}^{m}(\hat{x}), \\
\hat{x} \times(H(x) \times \hat{x})= \\
\frac{1}{k|x|} \sum_{n=1}^{\infty} \sum_{m=-n}^{n}\left(-i b_{n}^{m}\right)\left(\psi_{n}{ }^{\prime}(k|x|)+v_{n} \zeta_{n}^{(1)^{\prime}}(k|x|)\right) \operatorname{Grad} Y_{n}^{m}(\hat{x}) \\
+\frac{1}{k|x|} \sum_{n=1}^{\infty} \sum_{m=-n}^{n}\left(-a_{n}^{m}\right)\left(\psi_{n}(k|x|)+u_{n} \zeta_{n}^{(1)}(k|x|)\right)(\hat{x})\left(\hat{x} \times \operatorname{Grad}_{n}^{m}(\hat{x})\right)
\end{gathered}
$$

and finally, if $|x|=a$, we have

$$
\begin{gathered}
\hat{x} \times(E(x) \times \hat{x})+z_{r} \hat{x} \times H(x)= \\
\frac{1}{k|x|} \sum_{n=1}^{\infty} \sum_{m=-n}^{n} a_{n}^{m} A_{n}(k a)\left(\hat{x} \times \operatorname{Grad} Y_{n}^{m}(\hat{x})\right)-b_{n}^{m} B_{n}(k a) \operatorname{Grad} Y_{n}^{m}(\hat{x}),
\end{gathered}
$$

with

$$
\begin{aligned}
& A_{n}(k a)=\left(i \psi_{n}{ }^{\prime}(k a)+z_{r} \psi_{n}(k a)\right)+u_{n}\left(i \zeta_{n}^{(1)^{\prime}}(k a)+z_{r} \zeta_{n}^{(1)}(k a)\right) \\
& B_{n}(k a)=\left(\psi_{n}(k a)+i z_{r} \psi_{n}{ }^{\prime}(k a)\right)+v_{n}\left(\zeta_{n}^{(1)}(k a)+i z_{r} \zeta_{n}^{(1)^{\prime}}(k a)\right) .
\end{aligned}
$$

The boundary condition on the sphere leads to (43).

INRIA 


\section{A.2.4 Determination of the far field}

Lemma A.4 The far field is given by

$$
\begin{gathered}
E^{\infty}(\hat{x} ; \hat{d}, p)= \\
4 \pi \sum_{n=1}^{\infty} \sum_{m=-n}^{n}\left(u_{n}\left(\overline{U_{n}^{m}}(\hat{d}) \cdot p\right) U_{n}^{m}(\hat{x})+v_{n}\left(\overline{V_{n}^{m}}(\hat{d}) \cdot p\right) V_{n}^{m}(\hat{x})\right) .
\end{gathered}
$$

where $u_{n}$ and $v_{n}$ are given in (43).

Proof. From the scattered field, it is easy to get the far field. We have

$$
E^{s}(x ; \hat{d}, p)=\sum_{n=1}^{\infty} \sum_{m=-n}^{n} b_{n}^{m} v_{n} N_{n}^{m}(x)+\frac{i}{k} a_{n}^{m} u_{n} \operatorname{curl} N_{n}^{m}(x),
$$

and the far field is obtained using once again Theorem 6.26 page 175 in [3]

$$
E^{\infty}(\hat{x} ; \hat{d}, p)=-\frac{1}{i k} \sum_{m=-n}^{n}(-i)^{n} \sum_{n=1}^{\infty} a_{n}^{m} u_{n} \operatorname{Grad} Y_{n}^{m}(\hat{x})+b_{n}^{m} v_{n}\left(\hat{x} \times \operatorname{Grad}_{n}^{m}(\hat{x})\right)
$$

or, more explicitly

$$
\begin{aligned}
& E^{\infty}(\hat{x} ; \hat{d}, p)=\sum_{n=1}^{\infty} \sum_{m=-n}^{n} \frac{4 \pi u_{n}}{n(n+1)}\left(\operatorname{Grad} \overline{Y_{n}^{m}}(\hat{d}) \cdot p\right) \operatorname{Grad} Y_{n}^{m}(\hat{x}) \\
& +\sum_{n=1}^{\infty} \sum_{m=-n}^{n} \frac{4 \pi v_{n}}{n(n+1)}\left(\hat{d} \times \operatorname{Grad} \overline{Y_{n}^{m}}(\hat{d}) \cdot p\right)\left(\hat{x} \times \operatorname{Grad} Y_{n}^{m}(\hat{x})\right) .
\end{aligned}
$$

and the result follows from the definition of $U_{n}^{m}$ and $V_{n}^{m}$.

\section{A.3 An addition identity for the gradients of spherical harmonics}

Lemma A.5 Let $\hat{x}$ and $\hat{y}$ be two directions on the unit sphere. Let $n$ be a non negative integer, we have

$$
4 \pi \sum_{m=-n}^{n}\left|V_{n}^{m}(\hat{z}) \cdot q\right|^{2}=4 \pi \sum_{m=-n}^{n}\left|U_{n}^{m}(\hat{z}) \cdot q\right|^{2}=\frac{2 n+1}{2}\|\hat{z} \times q\|^{2} .
$$

Proof. It is known that

$$
(2 n+1) P_{n}(\hat{x} \cdot \hat{y})=4 \pi \sum_{m=-n}^{n} Y_{n}^{m}(\hat{x}) \overline{Y_{n}^{m}}(\hat{y}) .
$$


Let $p_{x}$ and $p_{y}$ be two vectors. Differentiating the relationship above, we get

$$
\begin{gathered}
(2 n+1)\left(\operatorname{Grad}_{\hat{x}}\left(\operatorname{Grad}_{\hat{y}} P_{n}(\hat{x} \cdot \hat{y}) \cdot p_{x}\right)\right) \cdot p_{y}= \\
4 \pi \sum_{m=-n}^{n} \operatorname{Grad}_{n}^{m}(\hat{x}) \cdot p_{x} \operatorname{Grad} \overline{Y_{n}^{m}}(\hat{y}) \cdot p_{y} .
\end{gathered}
$$

A direct computation shows that

$$
\begin{gathered}
\left(\operatorname{Grad}_{\hat{x}}\left(\operatorname{Grad}_{\hat{y}} P_{n}(\hat{x} \cdot \hat{y}) \cdot p_{x}\right)\right) \cdot p_{y}= \\
{\left[\left(p_{x} \cdot p_{y}\right)-\left(\hat{x} \cdot p_{y}\right)\left(\hat{x} \cdot p_{x}\right)-\left(\hat{y} \cdot p_{y}\right)\left(\hat{y} \cdot p_{x}\right)+(\hat{x} \cdot \hat{y})\left(\hat{y} \cdot p_{x}\right)\left(\hat{x} \cdot p_{y}\right)\right] P_{n}^{\prime}(\hat{x} \cdot \hat{y})} \\
+\left[( \hat { x } \cdot p _ { y } - ( \hat { x } \cdot \hat { y } ) ( \hat { y } \cdot p _ { y } ) ] \left[\left(\hat{y} \cdot p_{x}-(\hat{x} \cdot \hat{y})\left(\hat{x} \cdot p_{x}\right)\right] P_{n}^{\prime \prime}(\hat{x} \cdot \hat{y}) .\right.\right.
\end{gathered}
$$

Taking $\hat{x}=\hat{y}=\hat{z}$ and $p_{x}=p_{y}=q$, we get

$$
4 \pi \sum_{m=-n}^{n}\left|\operatorname{Grad} Y_{n}^{m}(\hat{z}) \cdot q\right|^{2}=(2 n+1)\left(\|q\|^{2}-(q \cdot \hat{z})^{2}\right) P_{n}^{\prime}(1)
$$

and, since $P_{n}^{\prime}(1)=\frac{n(n+1)}{2},[11]$ page 519

$$
4 \pi \sum_{m=-n}^{n}\left|\operatorname{Grad} Y_{n}^{m}(\hat{z}) \cdot q\right|^{2}=\frac{2 n+1}{2} n(n+1)\|\hat{z} \times q\|^{2} .
$$

In the same way, $\hat{x}=\hat{y}=\hat{z}$ and $p_{x}=p_{y}=q \times \hat{z}$ provides

$$
4 \pi \sum_{m=-n}^{n}\left|\left(\hat{z} \times \operatorname{Grad} Y_{n}^{m}(\hat{z})\right) \cdot q\right|^{2}=\frac{2 n+1}{2} n(n+1)\|\hat{z} \times q\|^{2} .
$$

In terms of the vector spherical harmonics, these equalities read

$$
4 \pi \sum_{m=-n}^{n}\left|V_{n}^{m}(\hat{z}) \cdot q\right|^{2}=4 \pi \sum_{m=-n}^{n}\left|U_{n}^{m}(\hat{z}) \cdot q\right|^{2}=\frac{2 n+1}{2}\|\hat{z} \times q\|^{2} .
$$

INRIA 


\section{Aknowledgement}

We would like to thank the SINES for providing us the possibility to use the SGI ORIGIN 2000 computer and to thank Patrick Laug (project gamma at INRIA) who help us to generate the teapot mesh.

$\mathrm{RR} \mathrm{n}^{\circ} 4665$ 


\section{References}

[1] D. Colton, H. Haddar, And P. Monk, The linear sampling method for solving the electromagnetic inverse scattering problem, SIAM J. Sci. Comp., to appear.

[2] D. Colton, A. KIRsch, A simple method for solving inverse scattering problems in the resonance region, Inverse Problems 12 (1996), pp 383-393.

[3] D. Colton and R. Kress, Inverse Acoustic and Electromagnetic Scattering Theory, no. 93 in Applied Mathematical Sciences, Springer-Verlag, New York, second ed., 1998.

[4] H. HADDAR AND P. MoNK, The linear sampling method for solving the electromagnetic inverse medium problem, Inverse Problems, 18 (2002), pp. 891-906.

[5] H. HADDAR, The interior transmission problem for anisotropic Maxwell's equations and its applications to the inverse problem, (to appear)

[6] A. KIRSCH, Characterization of the shape of a scattering obstacle using the spectral data of the far field operator. Inverse Problems, 14 (1998), no. 6, pp. 1489-1512.

[7] V.A. Monozov, Methods for solving incorrectly posed problems, Springer Verlag, 1984.

[8] J.C. NÉDÉLEC, Acoustic and Electromagnetic Equation. Integral Representation for Harmonic Problems, no. 144 in Applied Mathematical Sciences, Springer-Verlag, New York, 2001.

[9] S.M. Rao, D.R. Wilton and A.W. Glisson Electromagnetic Scattering by surfaces of arbitrary shape IEEE Transactions on Antennas and Propagation, AP 30 (1982), pp. 409-418.

[10] T.B.A.Senior and J.L.Volakis, Approximate Boundary Conditions in Electromagnetics, IEE Press:New York and London, 1995.

[11] J. Van Bladel, Electromagnetic Fields, A Summa Book, Hemisphere Publishing Corporation (1985). 


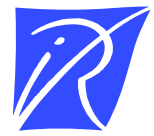

Unité de recherche INRIA Rocquencourt

Domaine de Voluceau - Rocquencourt - BP 105 - 78153 Le Chesnay Cedex (France)

Unité de recherche INRIA Futurs : Domaine de Voluceau - Rocquencourt - BP 105 - 78153 Le Chesnay Cedex (France) Unité de recherche INRIA Lorraine : LORIA, Technopôle de Nancy-Brabois - Campus scientifique 615, rue du Jardin Botanique - BP 101 - 54602 Villers-lès-Nancy Cedex (France)

Unité de recherche INRIA Rennes : IRISA, Campus universitaire de Beaulieu - 35042 Rennes Cedex (France)

Unité de recherche INRIA Rhône-Alpes : 655, avenue de l'Europe - 38330 Montbonnot-St-Martin (France)

Unité de recherche INRIA Sophia Antipolis : 2004, route des Lucioles - BP 93 - 06902 Sophia Antipolis Cedex (France)

INRIA - Domaine de Voluceau - Rocquencourt, BP 105 - 78153 Le Chesnay Cedex (France)

http://www.inria.fr

ISSN 0249-6399 